\title{
Effectiveness of plant-based repellents against different Anopheles species: a systematic review
}

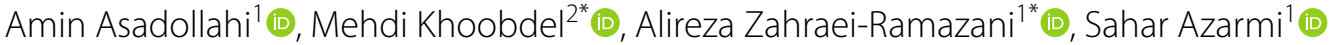 \\ and Sayed Hussain Mosawi ${ }^{3}$
}

\begin{abstract}
Plant-based repellents have been applied for generations in traditional practice as a personal protection approach against different species of Anopheles. Knowledge of traditional repellent plants is a significant resource for the development of new natural products as an alternative to chemical repellents. Many studies have reported evidence of repellant activities of plant extracts or essential oils against malaria vectors worldwide. This systematic review aimed to assess the effectiveness of plant-based repellents against Anopheles mosquitoes. All eligible studies on the repellency effects of plants against Anopheles mosquitoes published up to July 2018 were systematically searched through PubMed/Medline, Scopus and Google scholar databases. Outcomes measures were percentage repellency and protection time. A total of 62 trials met the inclusion criteria. The highest repellency effect was identified from Ligusticum sinense extract, followed by citronella, pine, Dalbergia sissoo, peppermint and Rhizophora mucronata oils with complete protection time ranging from 9.1 to $11.5 \mathrm{~h}$. Furthermore, essential oils from plants such as lavender, camphor, catnip, geranium, jasmine, broad-leaved eucalyptus, lemongrass, lemon-scented eucalyptus, amyris, narrow-leaved eucalyptus, carotin, cedarwood, chamomile, cinnamon oil, juniper, cajeput, soya bean, rosemary, niaouli, olive, tagetes, violet, sandalwood, litsea, galbanum, and Curcuma longa also showed good repellency with $8 \mathrm{~h}$ complete repellency against different species of Anopheles. Essential oils and extracts of some plants could be formulated for the development of eco-friendly repellents against Anopheles species. Plant oils may serve as suitable alternatives to synthetic repellents in the future as they are relatively safe, inexpensive, and are readily available in many parts of the world.
\end{abstract}

Keywords: Plant, Herb, Repellent, Repellency, Systematic review, Anopheles

\section{Background}

Mosquito-transmitted diseases remain a main source of illness and death [1]. Despite decades of malaria control efforts, malaria continues to be a major worldwide public health issue with 3.3 billion persons at risk in 106 countries and territories in the tropical and subtropical areas [2]. It

\footnotetext{
*Correspondence: khoobdel@yahoo.com; alirezazahraei@yahoo.com ${ }^{1}$ Department of Medical Entomology and Vector Control, School of Public Health, Tehran University of Medical Sciences, Tehran, Iran

${ }^{2}$ Health Research Centre, Lifestyle Institute, Baqiyatallah University of Medical Sciences, Tehran, Iran

Full list of author information is available at the end of the article
}

is one of the significant reasons for maternal and childhood morbidity and mortality, including low birth weight, stillbirths, and early infant death in sub-Saharan Africa [3]. Among 500 species of Anopheles mosquitoes known globally, more than 50 species can transmit malaria from the bite of the infected female Anopheles spp. [4]. Presently, there is no effective prophylactic anti-malarial vaccine and no suitable preventive measure other than vector control is available [5]. Thus, protection from mosquito bites is one of the best approaches to reduce the disease incidence.

The use of repellents to protect people from bites of mosquitoes previously has been acknowledged as part

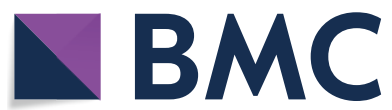

(c) The Author(s) 2019. This article is licensed under a Creative Commons Attribution 4.0 International License, which permits use, sharing, adaptation, distribution and reproduction in any medium or format, as long as you give appropriate credit to the original author(s) and the source, provide a link to the Creative Commons licence, and indicate if changes were made. The images or other third party material in this article are included in the article's Creative Commons licence, unless indicated otherwise in a credit line to the material. If material is not included in the article's Creative Commons licence and your intended use is not permitted by statutory regulation or exceeds the permitted use, you will need to obtain permission directly from the copyright holder. To view a copy of this licence, visit http://creativeco mmons.org/licenses/by/4.0/. The Creative Commons Public Domain Dedication waiver (http://creativecommons.org/publicdomain/ zero/1.0/) applies to the data made available in this article, unless otherwise stated in a credit line to the data. 
of an overall integrated insect-borne disease control programme [6]. Most commercial repellents are produced by using chemical components such as $\mathrm{N}, \mathrm{N}$-diethyl-metatoluamide (DEET), Allethrin, N, N-diethyl mendelic acid amide, and Dimethyl phthalate [1]. It has been identified that chemical repellents are not safe for public health and should be used with caution because of their detrimental impacts on synthetic fabric and plastic as well as toxic reactions, such as allergy, dermatitis, and cardiovascular and neurological side effects, which have been reported generally after misapplication [4]. The frequent use of synthetic repellents with chemical origin for mosquito control has disturbed natural ecosystems and resulted in the development of resistance to insecticides, resurgence in mosquito populations, and adverse impact on nontarget organisms [4, 7]. Accordingly, the idea of using natural mosquito repellent products as an alternative to develop new eco-friendly repellents could be an amicable solution to scale back the undesirable effects on environment and human health.

In recent years, interest in plant-based repellents has been revived, as they contain a rich source of bioactive phytochemicals that are safe and biodegradable into nontoxic by-products, which could be screened for insecticidal activities and mosquito repellent. Many studies have reported evidence of repellant activities of plant extracts or essential oils against malaria vectors around the world. The present systematic review was performed to reveal which plant-based repellent can be relied on to provide a prolonged and predictable protection from species of Anopheles mosquitoes without causing side effects on human health.

For this systematic review, all eligible studies on the repellency effects of plant-based repellants against Anopheles spp. published up to July 2018 were systematically searched through electronic databases PubMed, MEDLINE, Web of Science, Literature retrieval System of the Armed Forces Pest Management Board, Scopus and Google Scholar using the following Medical Subject Headings (Mesh) and keywords: (((Plant [Title/Abstract]) OR Plants [Title/Abstract]) OR herbal [Title/Abstract]) AND (botanical [Title/Abstract]) AND ((extract [Title/ Abstract]) OR extracts [Title/Abstract]) AND (("essential oil" [Title/Abstract]) OR "essential oils" [Title/Abstract]) AND ((((“'Insect repellent" [Mesh]) OR repellents) OR repellent) OR repellence) OR repellency) AND ((“Anopheles" [Mesh]) OR "Anopheles" [Title/Abstract]). The search was limited to English publications. In addition, a manual search was conducted to identify further pertinent articles using references from retrieved studies.

\section{Eligibility criteria}

Studies were included in the present systematic review if they met these criteria: (i) full-text publication was written in English, (ii) inspected the repellency effects of plant extracts and essential oils against malaria vectors, Anopheles spp. mosquitoes, and, (iii) reported the percentage of repellency or complete protection time. Following studies were excluded: studies exploring the repellency effect of chemical-based products, studies examining the repellency effect of animal extracts, animal studies (studies not on human subjects), articles without full texts, reviews, duplicate articles, abstracts, republished data, comments, conference papers, editorials, and studies with insufficient data. In addition, studies were excluded if the information could not be extracted. A screening of titles and abstracts followed by a full-text review was performed by two investigators. All titles and abstracts were screened by two independent investigators for eligibility. If a consensus was reached, a study was excluded or selected to full-text screening. If a consensus was not reached, another reviewer was consulted to resolve any feasible discrepancies.

\section{Data extraction}

After identifying the eligible studies, the following data were collected from each study by application of standardized data collection form to improve accuracy and critical appraisal: the first author name, country of origin, journal details, publication year, condition of study (field or laboratory), plant name, Anopheles species, concentration or dose of repellents, repellency percentage and complete protection time. All data were independently extracted by two reviewers and disagreements were solved by discussion, and if necessary, a third author was involved.

A total of 383 studies were found by the initial literature search of the databases. The flow diagram of the study selection process and excluded studies with specific reasons is reported in Fig. 1. Of the 324 excluded citations, 102 were duplicated studies; 149 were not relevant to the repellency effect of plants on Anopheles spp. after screening titles/abstracts; 11 were review publications; 8 investigated the repellency impact of chemical-based repellents or animal extracts; 7 studies were conducted on laboratory animals; 12 were abstracts, conference papers, comments, and editorials; 10 studies had not reported sufficient data regarding the percentage of repellency or complete protection time; and, 15 studies were other irrelevant studies. The primary eligibility process yielded 59 documents and crosscheck of the references of reviews and other databases search provided 3 further articles [8-10]. A total of 62 studies conducted in different countries, including India [7-40], Thailand [4, 


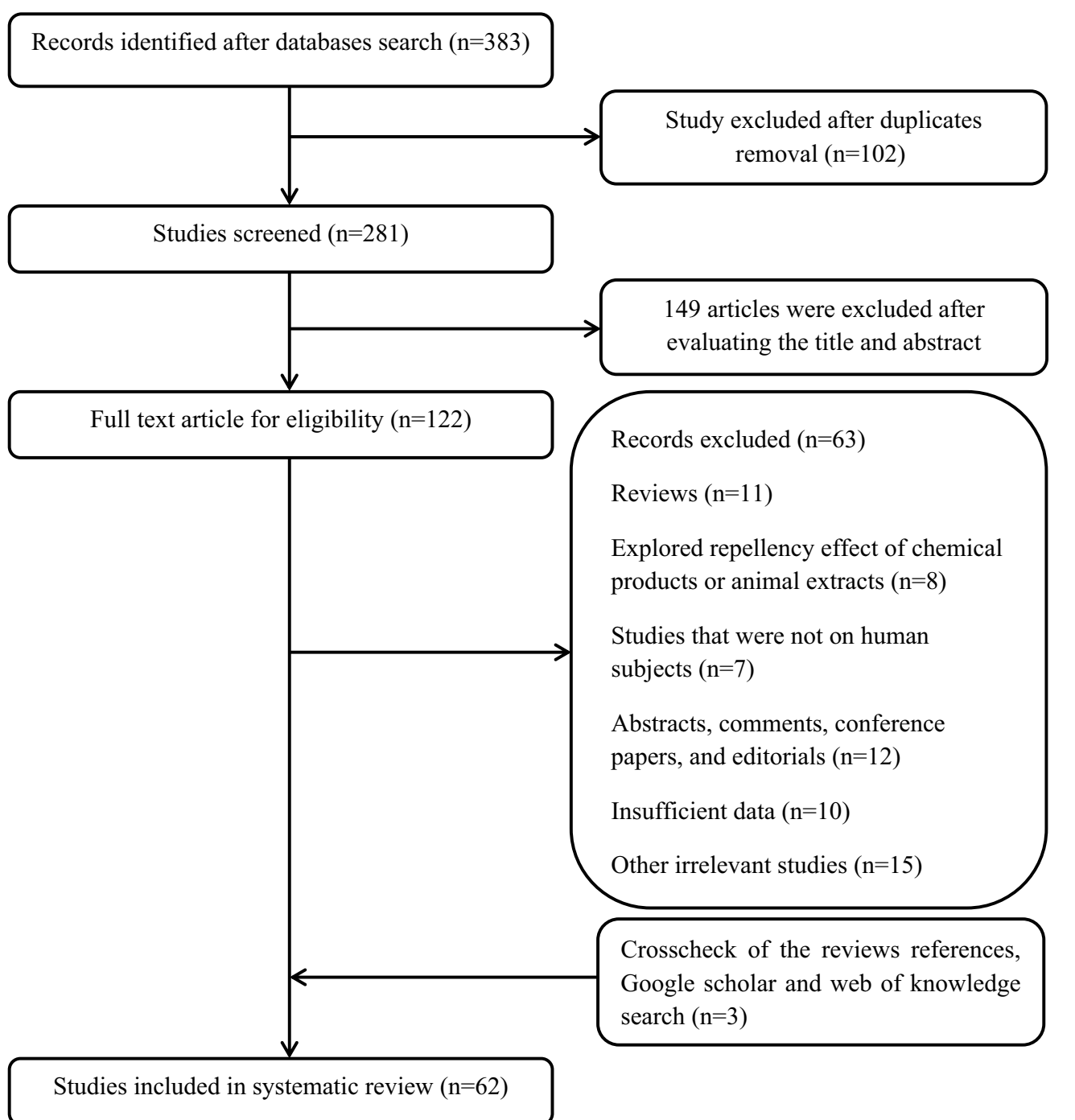

Fig. 1 Study selection process, up to July 2018

5, 41-48], Ethiopia [49-52], Kenya [53-57], Germany [6], Nigeria [1], USA [58], Tanzania [59], Brazil [60], Sudan [61], Iran [62], Cameron [63] and Ivory Coast [64] were eventually included in the systematic review based on the inclusion criteria for the effect of plantbased repellents on species of Anopheles mosquitoes. The included studies were published between 1999 and 2018. Expect for 6 studies which were field trial, other studies were conducted on laboratory condition. None of the studies reported the inclusion and exclusion criteria explicitly other than specifying a healthy volunteer. Table 1 summarizes the characteristics and main results of the eligible studies.

\section{Effectiveness of plant-based products against Anopheles}

spp.

Potential plant-based repellents stratified by protection time with at least $4 \mathrm{~h}$ protection time are reported in Table 2. The highest repellency effect was identified from Ligusticum sinense extract, followed by citronella, pine, Dalbergia sissoo, peppermint and Rhizophora mucronata oils with complete protection time ranging from 9.1 to $11.5 \mathrm{~h}$. Ethanolic $25 \%$ extract of $L$. sinense was able to completely repel Anopheles minimus for $11.5 \mathrm{~h}$. Furthermore, essential oils from plants such as lavender, camphor, catnip, geranium, jasmine, broad-leaved 
Table 1 Characteristics of studies. Characteristics of studies included in the systematic review, up to July 2018

\begin{tabular}{|c|c|c|c|c|c|c|c|c|}
\hline Study & Year & Country & Study type & $\begin{array}{l}\text { Plant extract/ } \\
\text { (essential oil) }\end{array}$ & $\begin{array}{l}\text { Concentration } \\
\text { dose }\end{array}$ & Anopheles species & Repellency \% & $\begin{array}{l}\text { Protection } \\
\text { time } \\
\text { (hours) }\end{array}$ \\
\hline \multirow[t]{2}{*}{ Ansari et al. } & 2005 & India & Field & Pine oil (Pinus) & $\begin{array}{l}1 \mathrm{ml} \text { without dilu- } \\
\text { tion }\end{array}$ & An. culicifacies & 100 & 11 \\
\hline & & & & $\begin{array}{l}\text { Citronella (lemon- } \\
\text { grass oil) }\end{array}$ & $\begin{array}{l}1 \mathrm{ml} \text { without dilu- } \\
\text { tion }\end{array}$ & An. culicifacies & 100 & 11 \\
\hline \multirow[t]{3}{*}{ Ansari et al. } & 2000 & India & Field & D. sissoo oil & $\begin{array}{l}1 \mathrm{ml} \text { without dilu- } \\
\text { tion }\end{array}$ & An. culicifacies & 96.1 & 10.3 \\
\hline & & & & D. sissoo oil & $\begin{array}{l}1 \mathrm{ml} \text { without dilu- } \\
\text { tion }\end{array}$ & An. annularis & 100 & 11 \\
\hline & & & & D. sissoo oil & $\begin{array}{l}1 \mathrm{ml} \text { without dilu- } \\
\text { tion }\end{array}$ & An. subpictus & 89.7 & 8 \\
\hline \multirow[t]{3}{*}{ Ansari et al. } & 2000 & India & Field & Peppermint oil & $\begin{array}{l}1 \mathrm{ml} \text { without dilu- } \\
\text { tion }\end{array}$ & An. culicifacies & 92.3 & 9.6 \\
\hline & & & & Peppermint oil & $\begin{array}{l}1 \mathrm{ml} \text { without dilu- } \\
\text { tion }\end{array}$ & An. annularis & 100 & 11 \\
\hline & & & & Peppermint oil & $\begin{array}{l}1 \mathrm{ml} \text { without dilu- } \\
\text { tion }\end{array}$ & An. subpictus & 83.1 & 7.3 \\
\hline \multirow[t]{14}{*}{ Amer et al. } & 2006 & Germany & Laboratory & $\begin{array}{l}\text { Citronella (Cym- } \\
\text { bopogon winteri- } \\
\text { anus) essential oils }\end{array}$ & $20 \%$ oil solutions & An. stephensi & 52.4 & 8 \\
\hline & & & & $\begin{array}{l}\text { Rosewood (Aniba } \\
\text { rosaeodora) essen- } \\
\text { tial oils }\end{array}$ & $20 \%$ oil solutions & An. stephensi & 4.8 & 6.5 \\
\hline & & & & $\begin{array}{l}\text { Lavender (Lavan- } \\
\text { dula angustifolia) } \\
\text { essential oils }\end{array}$ & $20 \%$ oil solutions & An. stephensi & 80.9 & 8 \\
\hline & & & & $\begin{array}{l}\text { Camphor (C. cam- } \\
\text { phora) essential } \\
\text { oils }\end{array}$ & $20 \%$ oil solutions & An. stephensi & 42.8 & 8 \\
\hline & & & & $\begin{array}{l}\text { Catnip (N. cataria) } \\
\text { essential oils }\end{array}$ & $20 \%$ oil solutions & An. stephensi & 100 & 8 \\
\hline & & & & $\begin{array}{l}\text { Geranium (Pelargo- } \\
\text { nium graveolens) } \\
\text { essential oils }\end{array}$ & $20 \%$ oil solutions & An. stephensi & 61.9 & 8 \\
\hline & & & & $\begin{array}{l}\text { Thyme (T. serpyllum) } \\
\text { essential oils }\end{array}$ & $20 \%$ oil solutions & An. stephensi & 33.3 & 7.5 \\
\hline & & & & $\begin{array}{l}\text { Eucalyptus (E. globu- } \\
\text { lus) essential oils }\end{array}$ & $20 \%$ oil solutions & An. stephensi & 28.6 & 5.5 \\
\hline & & & & $\begin{array}{l}\text { Jasmine (Jasminum } \\
\text { grandiflorum) } \\
\text { essential oils }\end{array}$ & $20 \%$ oil solutions & An. stephensi & 100 & 8 \\
\hline & & & & $\begin{array}{l}\text { Broad-leaved euca- } \\
\text { lyptus (Eucalyptus } \\
\text { dives) essential oils }\end{array}$ & $20 \%$ oil solutions & An. stephensi & 38.1 & 8 \\
\hline & & & & $\begin{array}{l}\text { Lemongrass (Cym- } \\
\text { bopogon citratus) } \\
\text { essential oil }\end{array}$ & $20 \%$ oil solutions & An. stephensi & 100 & 8 \\
\hline & & & & $\begin{array}{l}\text { Lemon-scented } \\
\text { eucalyptus (E. } \\
\text { citriodora) essen- } \\
\text { tial oil }\end{array}$ & $20 \%$ oil solutions & An. stephensi & 52.4 & 8 \\
\hline & & & & $\begin{array}{l}\text { Fichtennadel (Picea } \\
\text { excelsa) essential } \\
\text { oil }\end{array}$ & $20 \%$ oil solutions & An. stephensi & 19 & 3 \\
\hline & & & & $\begin{array}{c}\text { Amyris (Amyris } \\
\text { balsamifera) } \\
\text { essential oil }\end{array}$ & $20 \%$ oil solutions & An. stephensi & 100 & 8 \\
\hline
\end{tabular}


Table 1 (continued)

\begin{tabular}{|c|c|c|c|c|c|c|c|c|}
\hline Study & Year & Country & Study type & $\begin{array}{l}\text { Plant extract/ } \\
\text { (essential oil) }\end{array}$ & $\begin{array}{l}\text { Concentration } \\
\text { dose }\end{array}$ & Anopheles species & Repellency \% & $\begin{array}{l}\text { Protection } \\
\text { time } \\
\text { (hours) }\end{array}$ \\
\hline & & & & $\begin{array}{l}\text { Lemon (Citrus limon) } \\
\text { essential oil }\end{array}$ & $20 \%$ oil solutions & An. stephensi & 9.5 & 7 \\
\hline & & & & $\begin{array}{l}\text { Narrow-leaved } \\
\text { eucalyptus (Euca- } \\
\text { lyptus radiata) } \\
\text { essential oil }\end{array}$ & $20 \%$ oil solutions & An. stephensi & 42.8 & 8 \\
\hline & & & & $\begin{array}{l}\text { Carotin oil (Glycina } \\
\text { soja) essential oil }\end{array}$ & $20 \%$ oil solutions & An. stephensi & 9.5 & 8 \\
\hline & & & & $\begin{array}{l}\text { Cedarwood (Juni- } \\
\text { perus virginiana) } \\
\text { essential oil }\end{array}$ & $20 \%$ oil solutions & An. stephensi & 38.1 & 8 \\
\hline & & & & $\begin{array}{l}\text { frankincense } \\
\text { (Boswellia carteri) } \\
\text { essential oil }\end{array}$ & $20 \%$ oil solutions & An. stephensi & 19 & 5 \\
\hline & & & & $\begin{array}{l}\text { Dill (Anethum gra- } \\
\text { veolens) essential } \\
\text { oil }\end{array}$ & $20 \%$ oil solutions & An. stephensi & 71.4 & 3.5 \\
\hline & & & & $\begin{array}{l}\text { Myrtle (M. commu- } \\
\text { nis) essential oil }\end{array}$ & $20 \%$ oil solutions & An. stephensi & 42.8 & 6.5 \\
\hline & & & & $\begin{array}{l}\text { Chamomile } \\
\text { (Anthemis nobilis) } \\
\text { essential oil }\end{array}$ & $20 \%$ oil solutions & An. stephensi & 76.2 & 8 \\
\hline & & & & $\begin{array}{l}\text { Cinnamon (C. zey- } \\
\text { lanicum) essential } \\
\text { oil }\end{array}$ & $20 \%$ oil solutions & An. stephensi & 100 & 8 \\
\hline & & & & $\begin{array}{l}\text { Juniper (Juniperus } \\
\text { communis) essen- } \\
\text { tial oil }\end{array}$ & $20 \%$ oil solutions & An. stephensi & 76.2 & 8 \\
\hline & & & & $\begin{array}{l}\text { Sage (Salvia sclarea) } \\
\text { essential oil }\end{array}$ & $20 \%$ oil solutions & An. stephensi & 19 & 5 \\
\hline & & & & $\begin{array}{l}\text { Peppermint } \\
\text { (Mentha piperita) } \\
\text { essential oil }\end{array}$ & $20 \%$ oil solutions & An. stephensi & 57.1 & 6.5 \\
\hline & & & & $\begin{array}{l}\text { Basil (Ocimum basili- } \\
\text { cum) essential oil }\end{array}$ & $20 \%$ oil solutions & An. stephensi & 66.7 & 3.5 \\
\hline & & & & $\begin{array}{l}\text { Cajeput (Melaleuca } \\
\text { leucadendron) } \\
\text { essential oil }\end{array}$ & $20 \%$ oil solutions & An. stephensi & 100 & 8 \\
\hline & & & & $\begin{array}{c}\text { Soya bean (Glycina } \\
\text { max) essential oil }\end{array}$ & $20 \%$ oil solutions & An. stephensi & 76.2 & 8 \\
\hline & & & & $\begin{array}{l}\text { Rosemary (R. offici- } \\
\text { nalis) essential oil }\end{array}$ & $20 \%$ oil solutions & An. stephensi & 100 & 8 \\
\hline & & & & $\begin{array}{l}\text { Niaouli (Melaleuca } \\
\text { quinquenervia) } \\
\text { essential oil }\end{array}$ & $20 \%$ oil solutions & An. stephensi & 100 & 8 \\
\hline & & & & $\begin{array}{l}\text { Olive (O. europaea) } \\
\text { essential oil }\end{array}$ & $20 \%$ oil solutions & An. stephensi & 71.4 & 8 \\
\hline & & & & $\begin{array}{l}\text { Black pepper (Piper } \\
\text { nigrum) essential } \\
\text { oil }\end{array}$ & $20 \%$ oil solutions & An. stephensi & 61.9 & 3 \\
\hline & & & & $\begin{array}{l}\text { Verbena (Lippia } \\
\text { citriodora) essen- } \\
\text { tial oil }\end{array}$ & $20 \%$ oil solutions & An. stephensi & 38.1 & 5.5 \\
\hline & & & & $\begin{array}{l}\text { tagetes (T. minuta) } \\
\text { essential oil }\end{array}$ & $20 \%$ oil solutions & An. stephensi & 100 & 8 \\
\hline & & & & $\begin{array}{l}\text { Violet (Viola odorata) } \\
\text { essential oil }\end{array}$ & $20 \%$ oil solutions & An. stephensi & 100 & 8 \\
\hline
\end{tabular}


Table 1 (continued)

\begin{tabular}{|c|c|c|c|c|c|c|c|c|}
\hline Study & Year & Country & Study type & $\begin{array}{l}\text { Plant extract/ } \\
\text { (essential oil) }\end{array}$ & $\begin{array}{l}\text { Concentration } \\
\text { dose }\end{array}$ & Anopheles species & Repellency \% & $\begin{array}{l}\text { Protection } \\
\text { time } \\
\text { (hours) }\end{array}$ \\
\hline & & & & $\begin{array}{l}\text { Sandalwood } \\
\text { (Santalum album) } \\
\text { essential oil }\end{array}$ & $20 \%$ oil solutions & An. stephensi & 100 & 8 \\
\hline & & & & $\begin{array}{l}\text { Litsea (Litsea } \\
\text { cubeba) Essential } \\
\text { oil }\end{array}$ & $20 \%$ oil solutions & An. stephensi & 100 & 8 \\
\hline & & & & $\begin{array}{l}\text { Helichrysum (Heli- } \\
\text { chrysum italicum) } \\
\text { essential oil }\end{array}$ & $20 \%$ oil solutions & An. stephensi & 47.6 & 6 \\
\hline & & & & $\begin{array}{l}\text { Galbanum (Ferula } \\
\text { galbaniflua) } \\
\text { essential oil }\end{array}$ & $20 \%$ oil solutions & An. stephensi & 100 & 8 \\
\hline & & & & $\begin{array}{l}\text { Chamomile } \\
\text { (Chamaemelum } \\
\text { nobile) essential } \\
\text { oil }\end{array}$ & $20 \%$ oil solutions & An. stephensi & 47.6 & 5.5 \\
\hline Amerasan et al. & 2012 & India & Laboratory & $\begin{array}{l}\text { Cassia tora Linn } \\
\text { methanol extract }\end{array}$ & $\begin{array}{l}1 \mathrm{mg} / \mathrm{cm}^{2} \\
2.5 \mathrm{mg} / \mathrm{cm}^{2} \\
5.0 \mathrm{mg} / \mathrm{cm}^{2}\end{array}$ & An. stephensi & $\begin{array}{l}100 \\
100 \\
100\end{array}$ & $\begin{array}{l}2 \\
2 \\
2.5\end{array}$ \\
\hline \multirow[t]{2}{*}{ Abiy et al. } & 2015 & Ethiopia & Field & $20 \%$ neem oil & $\begin{array}{l}\text { Neem and china- } \\
\text { berry oils were } \\
\text { diluted to 20\% } \\
\text { using Niger seed } \\
\text { (noog abyssinia) } \\
\text { oil }\end{array}$ & An. arabiensis & 71 & 3 \\
\hline & & & & $20 \%$ chinaberry oil & $\begin{array}{l}\text { Neem and china- } \\
\text { berry oils were } \\
\text { diluted to } 20 \% \\
\text { using Niger seed } \\
\text { (noog abyssinia) } \\
\text { oil }\end{array}$ & An. arabiensis & 70 & 1 \\
\hline \multirow[t]{5}{*}{ Alayo et al. } & \multirow[t]{5}{*}{2015} & \multirow[t]{5}{*}{ Nigeria } & \multirow[t]{5}{*}{ Laboratory } & \multirow{5}{*}{$\begin{array}{l}\text { Cassia mimosoides } \\
\text { petroleum ether } \\
\text { extract }\end{array}$} & Cream $0.5 \% \mathrm{w} / \mathrm{w}$ & \multirow[t]{5}{*}{ An.gambiae } & 48 & - \\
\hline & & & & & Cream 1\% w/w & & 88 & - \\
\hline & & & & & Cream 2\% w/w & & 100 & 0.08 \\
\hline & & & & & Cream 4\% w/w & & 100 & 0.08 \\
\hline & & & & & Cream 6\% w/w & & 100 & 0.08 \\
\hline Alwala et al. & 2010 & Kenya & Laboratory & $\begin{array}{l}\text { Mangifera indica } \\
\text { essential Oil }\end{array}$ & $10 \%$ solution & An.gambiae & 100 & - \\
\hline Baskar et al. & 2018 & India & Laboratory & $\begin{array}{l}\text { Atalantia mono- } \\
\text { phylla essential oil }\end{array}$ & 50 ppm & An. stephensi & - & 6.85 \\
\hline \multirow[t]{2}{*}{ Govindarajan et al. } & \multirow[t]{2}{*}{2010} & \multirow[t]{2}{*}{ India } & \multirow[t]{2}{*}{ Laboratory } & \multirow{2}{*}{$\begin{array}{l}\text { Sida acuta Burm. F. } \\
\text { extract }\end{array}$} & $2.5 \mathrm{mg} / \mathrm{cm}^{2}$ & An. stephensi & 100 & 2.5 \\
\hline & & & & & $5 \mathrm{mg} / \mathrm{cm}^{2}$ & An. stephensi & 100 & 3 \\
\hline \multirow[t]{3}{*}{ Govindarajan et al. } & 2011 & India & Laboratory & $\begin{array}{l}\text { Ervatamia coronaria } \\
\text { extract }\end{array}$ & $\begin{array}{l}1 \mathrm{mg} / \mathrm{cm}^{2} \\
2.5 \mathrm{mg} / \mathrm{cm}^{2} \\
5 \mathrm{mg} / \mathrm{cm}^{2}\end{array}$ & $\begin{array}{l}\text { An. stephensi } \\
\text { An. stephensi } \\
\text { An. stephensi }\end{array}$ & $\begin{array}{l}100 \\
100 \\
100\end{array}$ & $\begin{array}{l}2.5 \\
3 \\
3.5\end{array}$ \\
\hline & & & & $\begin{array}{l}\text { Caesalpinia pulcher- } \\
\text { rima extract }\end{array}$ & $1 \mathrm{mg} / \mathrm{cm}^{2}$ & An. stephensi & 100 & 2 \\
\hline & & & & & $2.5 \mathrm{mg} / \mathrm{cm}^{2}$ & An. stephensi & 100 & 2.5 \\
\hline \multirow[t]{5}{*}{ Govindarajan et al. } & 2011 & India & Laboratory & & $5 \mathrm{mg} / \mathrm{cm}^{2}$ & An. stephensi & 100 & 3 \\
\hline & & & & & $\begin{array}{l}2.5 \mathrm{mg} / \mathrm{cm}^{2} \\
5 \mathrm{mg} / \mathrm{cm}^{2}\end{array}$ & $\begin{array}{l}\text { An. subpictus } \\
\text { An. subpictus }\end{array}$ & $\begin{array}{l}100 \\
100\end{array}$ & $\begin{array}{l}2 \\
2.5\end{array}$ \\
\hline & & & & $\begin{array}{l}\text { R. officinalis L. essen- } \\
\text { tial oil }\end{array}$ & $1 \mathrm{mg} / \mathrm{cm}^{2}$ & An. subpictus & 100 & 1 \\
\hline & & & & & $2.5 \mathrm{mg} / \mathrm{cm}^{2}$ & An. subpictus & 100 & 1 \\
\hline & & & & & $5 \mathrm{mg} / \mathrm{cm}^{2}$ & An. subpictus & 100 & 1.5 \\
\hline
\end{tabular}


Table 1 (continued)

\begin{tabular}{|c|c|c|c|c|c|c|c|c|}
\hline Study & Year & Country & Study type & $\begin{array}{l}\text { Plant extract/ } \\
\text { (essential oil) }\end{array}$ & $\begin{array}{l}\text { Concentration } \\
\text { dose }\end{array}$ & Anopheles species & Repellency \% & $\begin{array}{l}\text { Protection } \\
\text { time } \\
\text { (hours) }\end{array}$ \\
\hline & & & & C. citrates Stapf. & $1 \mathrm{mg} / \mathrm{cm}^{2}$ & An. subpictus & 100 & 1 \\
\hline & & & & essential oil & $2.5 \mathrm{mg} / \mathrm{cm}^{2}$ & An. subpictus & 100 & 1.5 \\
\hline & & & & & $5 \mathrm{mg} / \mathrm{cm}^{2}$ & An. subpictus & 100 & 2 \\
\hline & & & & C. zeylanicum L. & $1 \mathrm{mg} / \mathrm{cm}^{2}$ & An. subpictus & 100 & 1 \\
\hline & & & & essential oil & $2.5 \mathrm{mg} / \mathrm{cm}^{2}$ & An. subpictus & 100 & 1 \\
\hline & & & & & $5 \mathrm{mg} / \mathrm{cm}^{2}$ & An. subpictus & 100 & 1.5 \\
\hline \multirow[t]{3}{*}{ Govindarajan et al. } & \multirow[t]{3}{*}{2016} & \multirow[t]{3}{*}{ India } & \multirow[t]{3}{*}{ Laboratory } & \multirow{3}{*}{$\begin{array}{l}\text { Zingiber nimmonii } \\
\text { essential oil }\end{array}$} & $1 \mathrm{mg} / \mathrm{cm}^{2}$ & An. stephensi & 100 & 2 \\
\hline & & & & & $2 \mathrm{mg} / \mathrm{cm}^{2}$ & An. stephensi & 100 & 2.5 \\
\hline & & & & & $5 \mathrm{mg} / \mathrm{cm}^{2}$ & An. stephensi & 100 & 3 \\
\hline \multirow[t]{4}{*}{ Jeyabalan et al. } & \multirow[t]{4}{*}{2003} & \multirow[t]{4}{*}{ India } & \multirow[t]{4}{*}{ Laboratory } & \multirow[t]{4}{*}{ P. citrosa leaf extract } & $0.5 \%$ & An. stephensi & 36 & - \\
\hline & & & & & $1 \%$ & An. stephensi & 51 & - \\
\hline & & & & & $2 \%$ & An. stephensi & 78 & - \\
\hline & & & & & $4 \%$ & An. stephensi & 100 & - \\
\hline \multirow[t]{4}{*}{ Karunamoorthi et al. } & \multirow[t]{4}{*}{2008} & \multirow[t]{4}{*}{ Ethiopia } & \multirow[t]{4}{*}{ Laboratory } & $\begin{array}{l}\text { Woira (O. europaea) } \\
\text { smoke }\end{array}$ & $\begin{array}{l}\text { Burning of } 25 \mathrm{~g} \\
\text { of dried plant } \\
\text { materials }\end{array}$ & An. arabiensis & 79.7 & - \\
\hline & & & & $\begin{array}{l}\text { Tinjut (Ostostegia } \\
\text { integrifolia) smoke }\end{array}$ & $\begin{array}{l}\text { Burning of } 25 \mathrm{~g} \\
\text { of dried plant } \\
\text { materials }\end{array}$ & An. arabiensis & 90.1 & - \\
\hline & & & & $\begin{array}{l}\text { Wogert (Silene mac- } \\
\text { roserene) smoke }\end{array}$ & $\begin{array}{l}\text { Burning of } 25 \mathrm{~g} \\
\text { of dried plant } \\
\text { materials }\end{array}$ & An. arabiensis & 93.6 & - \\
\hline & & & & $\begin{array}{l}\text { Kebercho (Echinops } \\
\text { sp.) extract }\end{array}$ & $\begin{array}{l}\text { Burning of } 25 \mathrm{~g} \\
\text { of dried plant } \\
\text { materials }\end{array}$ & An. arabiensis & 92.4 & - \\
\hline \multirow[t]{4}{*}{ Karunamoorthi et al. } & \multirow[t]{4}{*}{2010} & \multirow[t]{4}{*}{ Ethiopia } & \multirow[t]{4}{*}{ Laboratory } & \multirow[t]{4}{*}{ C. citratus extract } & $1 \mathrm{mg} / \mathrm{cm}^{2}$ & An. arabiensis & 100 & 3.2 \\
\hline & & & & & $1.5 \mathrm{mg} / \mathrm{cm}^{2}$ & An. arabiensis & 100 & 4.4 \\
\hline & & & & & $2 \mathrm{mg} / \mathrm{cm}^{2}$ & An. arabiensis & 100 & 5.3 \\
\hline & & & & & $2.5 \mathrm{mg} / \mathrm{cm}^{2}$ & An. arabiensis & 100 & 6.3 \\
\hline \multirow[t]{3}{*}{ Govindarajan et al. } & \multirow[t]{3}{*}{2016} & \multirow[t]{3}{*}{ India } & \multirow[t]{3}{*}{ Laboratory } & Origanum scabrum & $1 \mathrm{mg} / \mathrm{cm}^{2}$ & An. stephensi & 100 & 2.5 \\
\hline & & & & & $2 \mathrm{mg} / \mathrm{cm}^{2}$ & An. stephensi & 100 & 3 \\
\hline & & & & & $5 \mathrm{mg} / \mathrm{cm}^{2}$ & An. stephensi & 100 & 3.5 \\
\hline Haldar et al. & 2014 & India & Laboratory & Ficus krishnae smoke & $30 \mathrm{mg} / \mathrm{l}$ smoked & An. stephensi & 18 & 0.16 \\
\hline & & & & & $60 \mathrm{mg} / \mathrm{l} \mathrm{smoked}$ & An. stephensi & 100 & 0.5 \\
\hline & & & & & 90 mg/l smoked & An. stephensi & 100 & 1 \\
\hline Auysawasdi et al. & 2015 & Thailand & Laboratory & Curcuma longa & $5 \%$ & An. dirus & 100 & 4 \\
\hline & & & & essential oil & $10 \%$ & An. dirus & 100 & 5 \\
\hline & & & & & $15 \%$ & An. dirus & 100 & 5.5 \\
\hline & & & & & $20 \%$ & An. dirus & 100 & 5.5 \\
\hline & & & & & $25 \%$ & An. dirus & 100 & 8 \\
\hline & & & & E. globulus essential & $5 \%$ & An. dirus & 100 & 1.7 \\
\hline & & & & oil & $10 \%$ & An. dirus & 100 & 2.3 \\
\hline & & & & & $15 \%$ & An. dirus & 100 & 3 \\
\hline & & & & & $20 \%$ & An. dirus & 100 & 3 \\
\hline & & & & & $25 \%$ & An. dirus & 100 & 3.4 \\
\hline & & & & Citrus aurantium & $5 \%$ & An. dirus & 100 & 1.8 \\
\hline & & & & essential oil & $10 \%$ & An. dirus & 100 & 2.9 \\
\hline & & & & & $15 \%$ & An. dirus & 100 & 2.9 \\
\hline & & & & & $20 \%$ & An. dirus & 100 & 3 \\
\hline & & & & & $25 \%$ & An. dirus & 100 & 3.5 \\
\hline
\end{tabular}


Table 1 (continued)

\begin{tabular}{|c|c|c|c|c|c|c|c|c|}
\hline Study & Year & Country & Study type & $\begin{array}{l}\text { Plant extract/ } \\
\text { (essential oil) }\end{array}$ & $\begin{array}{l}\text { Concentration } \\
\text { dose }\end{array}$ & Anopheles species & Repellency \% & $\begin{array}{l}\text { Protection } \\
\text { time } \\
\text { (hours) }\end{array}$ \\
\hline \multirow[t]{8}{*}{ Barnard et al. } & \multirow[t]{8}{*}{1999} & \multirow[t]{8}{*}{ USA } & \multirow[t]{8}{*}{ Laboratory } & \multirow[t]{4}{*}{ Clove essential oil } & $25 \%$ & An. albimanus & 100 & 1.25 \\
\hline & & & & & $50 \%$ & An. albimanus & 100 & 1.5 \\
\hline & & & & & $75 \%$ & An. albimanus & 100 & 2.26 \\
\hline & & & & & $100 \%$ & An. albimanus & 100 & 3.55 \\
\hline & & & & \multirow[t]{4}{*}{ Thyme essential oil } & $25 \%$ & An. albimanus & 100 & 0.75 \\
\hline & & & & & $50 \%$ & An. albimanus & 100 & 0.5 \\
\hline & & & & & $75 \%$ & An. albimanus & 100 & 1 \\
\hline & & & & & $100 \%$ & An. albimanus & 100 & 1.75 \\
\hline \multirow[t]{6}{*}{ Kweka et al. } & \multirow[t]{6}{*}{2008} & \multirow[t]{6}{*}{ Tanzania } & \multirow[t]{6}{*}{ Laboratory } & Citronella & $500 \mathrm{mg} / \mathrm{m}^{2}$ & An.gambiae & 81 & - \\
\hline & & & & $\begin{array}{l}\text { Ocimum suave } \\
\text { extract }\end{array}$ & $500 \mathrm{mg} / \mathrm{m}^{2}$ & An.gambiae & 81 & - \\
\hline & & & & $\begin{array}{l}\text { Ocimum kilimand- } \\
\text { scharicum extract }\end{array}$ & $500 \mathrm{mg} / \mathrm{m}^{2}$ & An.gambiae & 73 & - \\
\hline & & & & Citronella & $500 \mathrm{mg} / \mathrm{m}^{2}$ & An. arabiensis & 85 & - \\
\hline & & & & O. suave extract & $500 \mathrm{mg} / \mathrm{m}^{2}$ & An. arabiensis & 89 & - \\
\hline & & & & $\begin{array}{l}\text { O. kilimandschari- } \\
\text { cum extract }\end{array}$ & $500 \mathrm{mg} / \mathrm{m}^{2}$ & An. arabiensis & 75 & - \\
\hline \multirow[t]{3}{*}{ Kovendan et al. } & \multirow[t]{3}{*}{2012} & \multirow[t]{3}{*}{ India } & \multirow[t]{3}{*}{ Laboratory } & \multirow[t]{3}{*}{ A. alnifolia extract } & $1 \mathrm{mg} / \mathrm{cm}^{2}$ & An. stephensi & 100 & 2 \\
\hline & & & & & $3 \mathrm{mg} / \mathrm{cm}^{2}$ & An. stephensi & 100 & 2 \\
\hline & & & & & $5 \mathrm{mg} / \mathrm{cm}^{2}$ & An. stephensi & 100 & 2.5 \\
\hline \multirow[t]{3}{*}{ Krishnappa et al. } & \multirow[t]{3}{*}{2012} & \multirow[t]{3}{*}{ India } & \multirow[t]{3}{*}{ Laboratory } & \multirow{3}{*}{$\begin{array}{l}\text { A. digitata crude } \\
\text { extract }\end{array}$} & $2 \mathrm{mg} / \mathrm{cm}^{2}$ & An. stephensi & 100 & 3 \\
\hline & & & & & $4 \mathrm{mg} / \mathrm{cm}^{2}$ & An. stephensi & 100 & 3.5 \\
\hline & & & & & $6 \mathrm{mg} / \mathrm{cm}^{2}$ & An. stephensi & 100 & 3.5 \\
\hline \multirow[t]{3}{*}{ Naine et al. } & \multirow[t]{3}{*}{2014} & \multirow[t]{3}{*}{ India } & Laboratory & Streptomyces sp. & $1 \mathrm{mg} / \mathrm{cm}^{2}$ & An. stephensi & 100 & 2 \\
\hline & & & & VITJS4 extract & $3 \mathrm{mg} / \mathrm{cm}^{2}$ & An. stephensi & 100 & 2 \\
\hline & & & & & $6 \mathrm{mg} / \mathrm{cm}^{2}$ & An. stephensi & 100 & 2 \\
\hline Murugan et al. & 2012 & India & Laboratory & Orange peel extract & 50 ppm & An. stephensi & 99 & - \\
\hline & & & & & 150 ppm & An. stephensi & 100 & 0.5 \\
\hline & & & & & 250 ppm & An. stephensi & 100 & 0.05 \\
\hline & & & & & 350 ppm & An. stephensi & 100 & 1.5 \\
\hline & & & & & 450 ppm & An. stephensi & 100 & 2 \\
\hline Padilha et al. & 2003 & Brazil & Field & Ocimum selloi oil & $10 \% \mathrm{v} / \mathrm{v}$ & An. braziliensis & 89 & 0.5 \\
\hline Konan et al. & 2003 & Ivory Coast & Laboratory & Karite nut butter oil & $75 \%$ & An. gambiae & 100 & 2 \\
\hline & & & & Palm oil & $75 \%$ & An.gambiae & 100 & 1.38 \\
\hline & & & & Coconut oil & $75 \%$ & An.gambiae & 100 & 0.76 \\
\hline Maheswaran et al. & 2013 & India & Laboratory & Confertifolin essen- & 0.62 ppm & An. stephensi & 100 & 1 \\
\hline & & & & tial oil & 1.25 ppm & An. stephensi & 100 & 2.5 \\
\hline & & & & & $2.5 \mathrm{ppm}$ & An. stephensi & 100 & 3 \\
\hline & & & & & 5 ppm & An. stephensi & 100 & 5 \\
\hline & & & & & 10 ppm & An. stephensi & 100 & 5.2 \\
\hline Panneerselvam et al. & 2013 & India & Laboratory & Andrographis pan- & $1 \mathrm{mg} / \mathrm{cm}^{2}$ & An. stephensi & 100 & 2 \\
\hline & & & & iculata methanol & $3 \mathrm{mg} / \mathrm{cm}^{2}$ & An. stephensi & 100 & 2.5 \\
\hline & & & & & $6 \mathrm{mg} / \mathrm{cm}^{2}$ & An. stephensi & 100 & 3 \\
\hline & & & & Cassia occidentalis & $1 \mathrm{mg} / \mathrm{cm}^{2}$ & An. stephensi & 100 & 2 \\
\hline & & & & $\begin{array}{l}\text { methanol leaf } \\
\text { extract }\end{array}$ & $3 \mathrm{mg} / \mathrm{cm}^{2}$ & An. stephensi & 100 & 2.5 \\
\hline & & & & & $6 \mathrm{mg} / \mathrm{cm}^{2}$ & An. stephensi & 100 & 2.5 \\
\hline
\end{tabular}


Table 1 (continued)

\begin{tabular}{|c|c|c|c|c|c|c|c|c|}
\hline Study & Year & Country & Study type & $\begin{array}{l}\text { Plant extract/ } \\
\text { (essential oil) }\end{array}$ & $\begin{array}{l}\text { Concentration } \\
\text { dose }\end{array}$ & Anopheles species & Repellency \% & $\begin{array}{l}\text { Protection } \\
\text { time } \\
\text { (hours) }\end{array}$ \\
\hline & & & & Euphorbia hirta & $1 \mathrm{mg} / \mathrm{cm}^{2}$ & An. stephensi & 100 & 2 \\
\hline & & & & methanol leaf & $3 \mathrm{mg} / \mathrm{cm}^{2}$ & An. stephensi & 100 & 2 \\
\hline & & & & & $6 \mathrm{mg} / \mathrm{cm}^{2}$ & An. stephensi & 100 & 2.5 \\
\hline \multirow[t]{5}{*}{ Panneerselvam et al. } & 2012 & India & Laboratory & Artemisia nilagirica & 50 ppm & An. stephensi & 95 & 0.5 \\
\hline & & & & & 150 ppm & An. stephensi & 98 & 0.5 \\
\hline & & & & & 250 ppm & An. stephensi & 100 & 0.5 \\
\hline & & & & & 350 ppm & An. stephensi & 100 & 1 \\
\hline & & & & & 450 ppm & An. stephensi & 100 & 2 \\
\hline \multirow[t]{21}{*}{ Phasomkusolsil et al. } & 2011 & Thailand & Laboratory & Cananga odorata oil & $0.02 \mathrm{mg} / \mathrm{cm}^{2}$ & An. dirus & 94 & - \\
\hline & & & & & $0.10 \mathrm{mg} / \mathrm{cm}^{2}$ & An. dirus & 92 & - \\
\hline & & & & & $0.21 \mathrm{mg} / \mathrm{cm}^{2}$ & An. dirus & 92 & - \\
\hline & & & & C. sinensis oil & $0.02 \mathrm{mg} / \mathrm{cm}^{2}$ & An. dirus & 40 & - \\
\hline & & & & & $0.10 \mathrm{mg} / \mathrm{cm}^{2}$ & An. dirus & 54 & - \\
\hline & & & & & $0.21 \mathrm{mg} / \mathrm{cm}^{2}$ & An. dirus & 84 & - \\
\hline & & & & C. citratus oil & $0.02 \mathrm{mg} / \mathrm{cm}^{2}$ & An. dirus & 76 & - \\
\hline & & & & & $0.10 \mathrm{mg} / \mathrm{cm}^{2}$ & An. dirus & 82 & - \\
\hline & & & & & $0.21 \mathrm{mg} / \mathrm{cm}^{2}$ & An. dirus & 98 & - \\
\hline & & & & Cymbopogon nardus & $0.02 \mathrm{mg} / \mathrm{cm}^{2}$ & An. dirus & 92 & - \\
\hline & & & & & $0.10 \mathrm{mg} / \mathrm{cm}^{2}$ & An. dirus & 92 & - \\
\hline & & & & & $0.21 \mathrm{mg} / \mathrm{cm}^{2}$ & An. dirus & 98 & - \\
\hline & & & & E. citriodora oil & $0.02 \mathrm{mg} / \mathrm{cm}^{2}$ & An. dirus & 52 & - \\
\hline & & & & & $0.10 \mathrm{mg} / \mathrm{cm}^{2}$ & An. dirus & 74 & - \\
\hline & & & & & $0.21 \mathrm{mg} / \mathrm{cm}^{2}$ & An. dirus & 86 & - \\
\hline & & & & O. basilicum oil & $0.02 \mathrm{mg} / \mathrm{cm}^{2}$ & An. dirus & 66 & - \\
\hline & & & & & $0.10 \mathrm{mg} / \mathrm{cm}^{2}$ & An. dirus & 74 & - \\
\hline & & & & & $0.21 \mathrm{mg} / \mathrm{cm}^{2}$ & An. dirus & 96 & - \\
\hline & & & & S. aromaticum oil & $0.02 \mathrm{mg} / \mathrm{cm}^{2}$ & An. dirus & 82 & - \\
\hline & & & & & $0.10 \mathrm{mg} / \mathrm{cm}^{2}$ & An. dirus & 92 & - \\
\hline & & & & & $0.21 \mathrm{mg} / \mathrm{cm}^{2}$ & An. dirus & 98 & - \\
\hline \multirow[t]{5}{*}{ Prabhu et al. } & 2011 & India & Laboratory & Moringa oleifera & $20 \%$ & An. stephensi & 23 & - \\
\hline & & & & extract & $40 \%$ & An. stephensi & 43 & - \\
\hline & & & & & $60 \%$ & An. stephensi & 58 & - \\
\hline & & & & & $80 \%$ & An. stephensi & 76 & - \\
\hline & & & & & $100 \%$ & An. stephensi & 90 & - \\
\hline \multirow[t]{12}{*}{ Rajkumar et al. } & 2007 & India & Laboratory & Centella asiatica & $2 \%$ & An. stephensi & - & 1 \\
\hline & & & & essential oil & $4 \%$ & An. stephensi & - & 1.78 \\
\hline & & & & & $6 \%$ & An. stephensi & - & 2.33 \\
\hline & & & & Ipomoea cairica & $2 \%$ & An. stephensi & - & 2.63 \\
\hline & & & & essential oil & $4 \%$ & An. stephensi & - & 4.13 \\
\hline & & & & & $6 \%$ & An. stephensi & - & 5.53 \\
\hline & & & & Momordica charan- & $2 \%$ & An. stephensi & - & 2.38 \\
\hline & & & & tia essential oil & $4 \%$ & An. stephensi & - & 3.93 \\
\hline & & & & & $6 \%$ & An. stephensi & - & 5.38 \\
\hline & & & & Psidium guajava & $2 \%$ & An. stephensi & - & 0.93 \\
\hline & & & & essential oil & $4 \%$ & An. stephensi & - & 1.48 \\
\hline & & & & & $6 \%$ & An. stephensi & - & 1.98 \\
\hline
\end{tabular}


Table 1 (continued)

\begin{tabular}{|c|c|c|c|c|c|c|c|c|}
\hline Study & Year & Country & Study type & $\begin{array}{l}\text { Plant extract/ } \\
\text { (essential oil) }\end{array}$ & $\begin{array}{l}\text { Concentration } \\
\text { dose }\end{array}$ & Anopheles species & Repellency \% & $\begin{array}{l}\text { Protection } \\
\text { time } \\
\text { (hours) }\end{array}$ \\
\hline & & & & Tridax procumbens & $2 \%$ & An. stephensi & - & 2.33 \\
\hline & & & & essential oil & $4 \%$ & An. stephensi & - & 3.78 \\
\hline & & & & & $6 \%$ & An. stephensi & - & 5.28 \\
\hline \multirow[t]{5}{*}{ Rajkumar et al. } & 2005 & India & Laboratory & Solanum trilobatum & $0.001 \%$ & An. stephensi & 100 & 1.15 \\
\hline & & & & & $0.005 \%$ & An. stephensi & 100 & 1.3 \\
\hline & & & & & $0.01 \%$ & An. stephensi & 100 & 1.51 \\
\hline & & & & & $0.015 \%$ & An. stephensi & 100 & 1.7 \\
\hline & & & & & $0.02 \%$ & An. stephensi & 100 & 2.03 \\
\hline \multirow[t]{3}{*}{ Rawani et al. } & 2012 & India & Laboratory & P. tuberosa extract & $1 \%$ & An. stephensi & 65 & 2.3 \\
\hline & & & & & $1.50 \%$ & An. stephensi & 80 & 4 \\
\hline & & & & & $2 \%$ & An. stephensi & 90 & 5 \\
\hline \multirow[t]{3}{*}{ Reegan et al. } & 2015 & India & Laboratory & Cliona celata extract & $1 \mathrm{mg} / \mathrm{cm}^{2}$ & An. stephensi & 100 & 1.08 \\
\hline & & & & & $2.5 \mathrm{mg} / \mathrm{cm}^{2}$ & An. stephensi & 100 & 1.71 \\
\hline & & & & & $5 \mathrm{mg} / \mathrm{cm}^{2}$ & An. stephensi & 100 & 1.21 \\
\hline \multirow[t]{3}{*}{ Swathi et al. } & 2012 & India & Laboratory & Datura stramonium & $0.1 \%$ & An. stephensi & - & 0.35 \\
\hline & & & & extract & $0.5 \%$ & An. stephensi & - & 0.72 \\
\hline & & & & & $1 \%$ & An. stephensi & - & 1.9 \\
\hline \multirow[t]{9}{*}{ Seyoum et al. } & 2002 & Kenya & Semi-field & Neem (A. indica) & $\begin{array}{l}\text { Periodic thermal } \\
\text { expulsion }\end{array}$ & An.gambiae & 24.5 & - \\
\hline & & & & $\begin{array}{l}\text { Lemon eucalyptus } \\
\text { (Corymbia citrio- } \\
\text { dora) }\end{array}$ & $\begin{array}{l}\text { Periodic thermal } \\
\text { expulsion }\end{array}$ & An. gambiae & 74.5 & - \\
\hline & & & & $\begin{array}{l}\text { Wild spikenard } \\
\text { (Hyptis suaveolens) }\end{array}$ & $\begin{array}{l}\text { Periodic thermal } \\
\text { expulsion }\end{array}$ & An. gambiae & -13.3 & - \\
\hline & & & & $\begin{array}{l}\text { Lantana (Lantana } \\
\text { camara) }\end{array}$ & $\begin{array}{l}\text { Periodic thermal } \\
\text { expulsion }\end{array}$ & An. gambiae & 42.4 & - \\
\hline & & & & $\begin{array}{l}\text { Fever tea (Lippia } \\
\text { uckambensis) }\end{array}$ & $\begin{array}{l}\text { Periodic thermal } \\
\text { expulsion }\end{array}$ & An. gambiae & 45.9 & - \\
\hline & & & & $\begin{array}{l}\text { Lime basil (Ocimum } \\
\text { americanum) }\end{array}$ & $\begin{array}{l}\text { Periodic thermal } \\
\text { expulsion }\end{array}$ & An.gambiae & 43.1 & - \\
\hline & & & & $\begin{array}{l}\text { Rican blue basil (O. } \\
\text { kilimandschari- } \\
\text { cum) }\end{array}$ & $\begin{array}{l}\text { Periodic thermal } \\
\text { expulsion }\end{array}$ & An. gambiae & 52.0 & - \\
\hline & & & & Tree basil (O. suave) & $\begin{array}{l}\text { Periodic thermal } \\
\text { expulsion }\end{array}$ & An.gambiae & 53.1 & - \\
\hline & & & & $\begin{array}{l}\text { Khaki weed ( } T \text {. } \\
\text { minuta) }\end{array}$ & $\begin{array}{l}\text { Placing branches } \\
\text { or whole plants } \\
\text { inside houses }\end{array}$ & An.gambiae & 54.8 & - \\
\hline Sanghong et al. & 2015 & Thailand & Laboratory & $\begin{array}{l}\text { L. sinense ethanolic } \\
\text { preparations }\end{array}$ & $25 \%$ & An. minimus & - & 11.5 \\
\hline Das et al. & 2003 & India & Laboratory & $\begin{array}{l}\text { Cymbopogan mar- } \\
\text { tinii martinii var } \\
\text { sofia oil }\end{array}$ & $\begin{array}{l}1 \mathrm{ml} \text { without dilu- } \\
\text { tion }\end{array}$ & An. sundaicus & 98 & 6 \\
\hline Nour et al. & 2009 & Sudan & Laboratory & $\begin{array}{l}\text { Basil (O. basilicum L.) } \\
\text { essential oil }\end{array}$ & $0.1 \mathrm{ml}$ & & 100 & 1.5 \\
\hline \multirow[t]{6}{*}{ Trongtokit et al. } & 2005 & Thailand & Laboratory & C. nardus essential & $10 \%$ & An. dirus & - & 0.66 \\
\hline & & & & & $50 \%$ & & - & 0.5 \\
\hline & & & & & $100 \%$ & & - & 1.16 \\
\hline & & & & P. cablin essential oil & $10 \%$ & An. dirus & - & 1.33 \\
\hline & & & & & $50 \%$ & & - & 2 \\
\hline & & & & & $100 \%$ & & - & 2.83 \\
\hline
\end{tabular}


Table 1 (continued)

\begin{tabular}{|c|c|c|c|c|c|c|c|c|}
\hline Study & Year & Country & Study type & $\begin{array}{l}\text { Plant extract/ } \\
\text { (essential oil) }\end{array}$ & $\begin{array}{l}\text { Concentration } \\
\text { dose }\end{array}$ & Anopheles species & Repellency \% & $\begin{array}{l}\text { Protection } \\
\text { time } \\
\text { (hours) }\end{array}$ \\
\hline & & & & \multirow{3}{*}{$\begin{array}{l}\text { Mullilam (Zanth- } \\
\text { oxylum limonella) } \\
\text { essential oil }\end{array}$} & $10 \%$ & \multirow[t]{3}{*}{ An. dirus } & - & 1 \\
\hline & & & & & $50 \%$ & & - & 2.16 \\
\hline & & & & & $100 \%$ & & - & 3.16 \\
\hline & & & & \multirow{3}{*}{$\begin{array}{l}\text { Clove (Syzygium } \\
\text { aromaticum) } \\
\text { essential oil }\end{array}$} & $10 \%$ & \multirow[t]{3}{*}{ An. dirus } & - & 1.33 \\
\hline & & & & & $50 \%$ & & - & 2.66 \\
\hline & & & & & $100 \%$ & & - & 3.5 \\
\hline \multirow[t]{4}{*}{ Yogananth et al. } & \multirow[t]{4}{*}{2015} & \multirow[t]{4}{*}{ India } & \multirow[t]{4}{*}{ Laboratory } & \multirow[t]{4}{*}{ R. mucronata oil } & $1 \mathrm{mg} / \mathrm{cm}^{2}$ & An. stephensi & 73 & 7.2 \\
\hline & & & & & $2 \mathrm{mg} / \mathrm{cm}^{2}$ & An. stephensi & 86 & 7.8 \\
\hline & & & & & $3 \mathrm{mg} / \mathrm{cm}^{2}$ & An. stephensi & 92 & 8.5 \\
\hline & & & & & $4 \mathrm{mg} / \mathrm{cm}^{2}$ & An. stephensi & 97 & 9.1 \\
\hline \multirow[t]{3}{*}{ Tawatsin et al. } & \multirow[t]{3}{*}{2000} & \multirow[t]{3}{*}{ Thailand } & \multirow[t]{3}{*}{ Laboratory } & $\begin{array}{l}\text { Turmeric (C. longa) } \\
\text { volatile oil }\end{array}$ & $3 \mathrm{ml}$ & An. dirus & 100 & 6 \\
\hline & & & & Citronella & $3 \mathrm{ml}$ & An. dirus & 100 & 6 \\
\hline & & & & Hairy basil oil & $3 \mathrm{ml}$ & An. dirus & 100 & 6 \\
\hline \multirow[t]{3}{*}{ Singh et al. } & \multirow[t]{3}{*}{2005} & \multirow[t]{3}{*}{ India } & \multirow[t]{3}{*}{ Laboratory } & \multirow{3}{*}{$\begin{array}{l}\text { Cyperus rotundus } \\
\text { Linn hexane } \\
\text { extract }\end{array}$} & $2.50 \%$ & An. stephensi & 95 & - \\
\hline & & & & & $5 \%$ & An. stephensi & 99 & - \\
\hline & & & & & $10 \%$ & An. stephensi & 100 & 6 \\
\hline \multirow[t]{3}{*}{ Mayeku et al. } & \multirow[t]{3}{*}{2013} & \multirow[t]{3}{*}{ Kenya } & \multirow[t]{3}{*}{ laboratory } & \multirow{3}{*}{$\begin{array}{l}\text { Conyza newii essen- } \\
\text { tial oil }\end{array}$} & $0.01 \mathrm{~g} / \mathrm{ml}$ & An.gambiae & 38 & - \\
\hline & & & & & $0.1 \mathrm{~g} / \mathrm{ml}$ & An. gambiae & 68 & - \\
\hline & & & & & $1 \mathrm{~g} / \mathrm{ml}$ & An.gambiae & 100 & - \\
\hline \multirow[t]{7}{*}{ Phasomkusolsil et al. } & \multirow[t]{7}{*}{2009} & Thailand & Laboratory & $\begin{array}{l}\text { Phlai (Z. cassumu- } \\
\text { nar) oil }\end{array}$ & $100 \mu \mathrm{l}$ & An. minimus & - & 2 \\
\hline & & & & $\begin{array}{l}\text { Turmeric (C. longa) } \\
\text { oil }\end{array}$ & $100 \mu \mathrm{l}$ & An. minimus & - & 1 \\
\hline & & & & $\begin{array}{l}\text { Mah-Khwuaen ( } Z \text {. } \\
\text { limonella) oil }\end{array}$ & $100 \mu \mathrm{l}$ & An. minimus & - & 0.66 \\
\hline & & & & $\begin{array}{l}\text { Citronella grass (C. } \\
\text { nardus) oil }\end{array}$ & $100 \mu l$ & An. minimus & - & 2.16 \\
\hline & & & & $\begin{array}{l}\text { Orange oil (Citrus } \\
\text { sinensis) oil }\end{array}$ & $100 \mu \mathrm{l}$ & An. minimus & - & 0.83 \\
\hline & & & & $\begin{array}{l}\text { Eucalyptus (E. citrio- } \\
\text { dora) oil }\end{array}$ & $100 \mu \mathrm{l}$ & An. minimus & - & 0.5 \\
\hline & & & & $\begin{array}{l}\text { Clove (S. aromati- } \\
\text { cum) oil }\end{array}$ & $100 \mu \mathrm{l}$ & An. minimus & - & 2 \\
\hline Trongtokit et al. & 2004 & Thailand & Laboratory & Clove oil & $20 \% \mathrm{gel}$ & An. dirus & - & 4.5 \\
\hline & & & & & cream $20 \%$ & An. dirus & - & 4.8 \\
\hline Birkett et al. & 2011 & Kenya & Laboratory & N. cataria & $0.01 \mathrm{mg}$ & An.gambiae & 17 & - \\
\hline & & & & & $0.1 \mathrm{mg}$ & An.gambiae & 97 & - \\
\hline & & & & & $1 \mathrm{mg}$ & An.gambiae & 100 & - \\
\hline Kamaraj et al. & 2011 & India & Laboratory & A. concinna extract & 500 ppm & An. stephensi & 21 & - \\
\hline Solomon et al. & 2012 & Ethiopia & Laboratory & Citronella extract & $20 \%$ & An. Arabiensis & 73 & - \\
\hline Soonwera et al. & 2015 & Thailand & Laboratory & C. odorata oil & $1 \%$ & An. dirus & 92 & - \\
\hline & & & & & $5 \%$ & An. dirus & 92 & - \\
\hline & & & & & $10 \%$ & An. dirus & 94 & - \\
\hline Sritabutra et al. & 2011 & Thailand & Laboratory & $\begin{array}{l}\text { Eucalyptus (E. glob- } \\
\text { ules) essencial oil }\end{array}$ & $0.1 \mathrm{ml}$ & An. dirus & - & 1.58 \\
\hline & & & & $\begin{array}{l}\text { Peppermint ( } M \text {. } \\
\text { piperita) essencial } \\
\text { oil }\end{array}$ & $0.1 \mathrm{ml}$ & An. dirus & - & 1.08 \\
\hline & & & & $\begin{array}{l}\text { Garlic (A. sativum) } \\
\text { essencial oil }\end{array}$ & $0.1 \mathrm{ml}$ & An. dirus & - & 0.68 \\
\hline
\end{tabular}


Table 1 (continued)

\begin{tabular}{|c|c|c|c|c|c|c|c|c|}
\hline Study & Year & Country & Study type & $\begin{array}{l}\text { Plant extract/ } \\
\text { (essential oil) }\end{array}$ & $\begin{array}{l}\text { Concentration } \\
\text { dose }\end{array}$ & Anopheles species & Repellency \% & $\begin{array}{l}\text { Protection } \\
\text { time } \\
\text { (hours) }\end{array}$ \\
\hline & & & & $\begin{array}{l}\text { Orange (C. sinensis) } \\
\text { essencial oil }\end{array}$ & $0.1 \mathrm{ml}$ & An. dirus & - & 0.83 \\
\hline & & & & $\begin{array}{l}\text { Citronella grass (C. } \\
\text { nardus) essencial } \\
\text { oil }\end{array}$ & $0.1 \mathrm{ml}$ & An. dirus & - & 0.8 \\
\hline & & & & $\begin{array}{l}\text { Lemongrass (C. } \\
\text { citratus) essencial } \\
\text { oil }\end{array}$ & $0.1 \mathrm{ml}$ & An. dirus & - & 1.63 \\
\hline & & & & $\begin{array}{l}\text { Clove (S. aromati- } \\
\text { cum) essencial oil }\end{array}$ & $0.1 \mathrm{ml}$ & An. dirus & - & 1 \\
\hline & & & & $\begin{array}{l}\text { Sweet basil ( } O \text {. } \\
\text { basilicum) essen- } \\
\text { cial oil }\end{array}$ & $0.1 \mathrm{ml}$ & An. dirus & - & 0.75 \\
\hline \multirow[t]{2}{*}{ Tavassoli et al. } & 2001 & iran & Laboratory & $\begin{array}{l}\text { Marigold (Calendula } \\
\text { officinalis) essen- } \\
\text { tial oil }\end{array}$ & $50 \%$ & An. stephensi & - & 2.15 \\
\hline & & & & Myrtle essential oil & $50 \%$ & An. stephensi & - & 4.36 \\
\hline \multirow[t]{6}{*}{ Younoussa et al. } & \multirow[t]{6}{*}{2016} & \multirow[t]{6}{*}{ Cameroon } & \multirow[t]{6}{*}{ Laboratory } & \multirow{3}{*}{$\begin{array}{l}\text { Annona senegalensis } \\
\text { leaf extract }\end{array}$} & $4.0 \mathrm{mg} / \mathrm{cm}^{2}$ & An. gambiae & - & 0.5 \\
\hline & & & & & $8.0 \mathrm{mg} / \mathrm{cm}^{2}$ & An.gambiae & - & 1 \\
\hline & & & & & $12.0 \mathrm{mg} / \mathrm{cm}^{2}$ & An.gambiae & - & 1.5 \\
\hline & & & & \multirow{3}{*}{$\begin{array}{l}\text { Boswellia dalzielii } \\
\text { leaf extract }\end{array}$} & $4.0 \mathrm{mg} / \mathrm{cm}^{2}$ & An.gambiae & 46 & \\
\hline & & & & & $8.0 \mathrm{mg} / \mathrm{cm}^{2}$ & An.gambiae & - & 0.5 \\
\hline & & & & & $12.0 \mathrm{mg} / \mathrm{cm}^{2}$ & An.gambiae & - & 1 \\
\hline \multirow[t]{3}{*}{ Govindarajan et al. } & \multirow[t]{3}{*}{2011} & \multirow[t]{3}{*}{ India } & \multirow[t]{3}{*}{ Laboratory } & \multirow{3}{*}{$\begin{array}{l}\text { Coccinia indica } \\
\text { extract }\end{array}$} & $1 \mathrm{mg} / \mathrm{cm}^{2}$ & An. stephensi & 100 & 3 \\
\hline & & & & & $2.5 \mathrm{mg} / \mathrm{cm}^{2}$ & An. stephensi & 100 & 3 \\
\hline & & & & & $5 \mathrm{mg} / \mathrm{cm}^{2}$ & An. stephensi & 100 & 3.5 \\
\hline \multirow[t]{3}{*}{ Govindarajan et al. } & \multirow[t]{3}{*}{2012} & \multirow[t]{3}{*}{ India } & \multirow[t]{3}{*}{ Laboratory } & \multirow{3}{*}{$\begin{array}{l}\text { Cardiospermum } \\
\text { halicacabum oil }\end{array}$} & $1 \mathrm{mg} / \mathrm{cm}^{2}$ & An. stephensi & 100 & 2 \\
\hline & & & & & $2.5 \mathrm{mg} / \mathrm{cm}^{2}$ & An. stephensi & 100 & 2.5 \\
\hline & & & & & $5 \mathrm{mg} / \mathrm{cm}^{2}$ & An. stephensi & 100 & 3 \\
\hline \multirow[t]{3}{*}{ Govindarajan et al. } & \multirow[t]{3}{*}{2014} & \multirow[t]{3}{*}{ India } & \multirow[t]{3}{*}{ Laboratory } & \multirow{3}{*}{$\begin{array}{l}\text { Asparagus racemo- } \\
\text { sus crude extract }\end{array}$} & $1 \mathrm{mg} / \mathrm{cm}^{2}$ & An. stephensi & 100 & 2.5 \\
\hline & & & & & $2 \mathrm{mg} / \mathrm{cm}^{2}$ & An. stephensi & 100 & 2.5 \\
\hline & & & & & $5 \mathrm{mg} / \mathrm{cm}^{2}$ & An. stephensi & 100 & 3 \\
\hline \multirow[t]{3}{*}{ Govindarajan et al. } & \multirow[t]{3}{*}{2015} & \multirow[t]{3}{*}{ India } & \multirow[t]{3}{*}{ Laboratory } & Delonix elata crude & $1 \mathrm{mg} / \mathrm{cm}^{2}$ & An. stephensi & 100 & 2.5 \\
\hline & & & & extract & $2.5 \mathrm{mg} / \mathrm{cm}^{2}$ & An. stephensi & 100 & 3 \\
\hline & & & & & $5 \mathrm{mg} / \mathrm{cm}^{2}$ & An. stephensi & 100 & 3.5 \\
\hline Innocent et al. & 2014 & Kenya & Laboratory & Uvariodendron gor- & $0.01 \mathrm{w} / \mathrm{v}$ & An.gambiae & 29 & - \\
\hline & & & & gonis essential oil & $0.1 \mathrm{w} / \mathrm{v}$ & An.gambiae & 48 & - \\
\hline & & & & & $1 \mathrm{w} / \mathrm{v}$ & An.gambiae & 57 & - \\
\hline & & & & & $10 \mathrm{w} / \mathrm{v}$ & An.gambiae & 64 & - \\
\hline & & & & Clausena anisata & $0.01 \mathrm{w} / \mathrm{v}$ & An.gambiae & 13 & - \\
\hline & & & & essential oil & $0.1 \mathrm{w} / \mathrm{v}$ & An.gambiae & 21 & - \\
\hline & & & & & $1 \mathrm{w} / \mathrm{v}$ & An.gambiae & 42 & - \\
\hline & & & & & $10 \mathrm{w} / \mathrm{v}$ & An.gambiae & 56 & - \\
\hline & & & & Lantana vibunoides & $0.01 \mathrm{w} / \mathrm{v}$ & An.gambiae & 26 & - \\
\hline & & & & essential oil & $0.1 \mathrm{w} / \mathrm{v}$ & An. gambiae & 46 & - \\
\hline & & & & & $1 \mathrm{w} / \mathrm{v}$ & An.gambiae & 54 & - \\
\hline & & & & & $10 \mathrm{w} / \mathrm{v}$ & An.gambiae & 62 & - \\
\hline
\end{tabular}


Table 1 (continued)

\begin{tabular}{|c|c|c|c|c|c|c|c|c|}
\hline Study & Year & Country & Study type & $\begin{array}{l}\text { Plant extract/ } \\
\text { (essential oil) }\end{array}$ & $\begin{array}{l}\text { Concentration } \\
\text { dose }\end{array}$ & Anopheles species & Repellency \% & $\begin{array}{l}\text { Protection } \\
\text { time } \\
\text { (hours) }\end{array}$ \\
\hline \multirow[t]{5}{*}{ Kumar et al. } & \multirow[t]{5}{*}{2012} & \multirow[t]{5}{*}{ India } & \multirow[t]{5}{*}{ Laboratory } & \multirow{5}{*}{$\begin{array}{l}\text { Sargassum wightii } \\
\text { Greville metha- } \\
\text { nolic extract }\end{array}$} & $2 \mathrm{mg} / \mathrm{l}$ & An. sundaicus & 26 & - \\
\hline & & & & & $4 \mathrm{mg} / \mathrm{l}$ & An. sundaicus & 40 & - \\
\hline & & & & & $6 \mathrm{mg} / \mathrm{l}$ & An. sundaicus & 57 & - \\
\hline & & & & & $8 \mathrm{mg} / \mathrm{l}$ & An. sundaicus & 71 & - \\
\hline & & & & & 10 mg/l & An. sundaicus & 89 & - \\
\hline \multirow{3}{*}{$\begin{array}{l}\text { Madhiyazhagan } \\
\text { et al. }\end{array}$} & \multirow[t]{3}{*}{2014} & \multirow[t]{3}{*}{ India } & \multirow[t]{3}{*}{ Laboratory } & \multirow[t]{3}{*}{ O. canum extract } & $0.49 \mathrm{mg} / \mathrm{l}$ & An. stephensi & 63 & - \\
\hline & & & & & $0.99 \mathrm{mg} / \mathrm{l}$ & An. stephensi & 77 & - \\
\hline & & & & & 1.99 mg/l & An. stephensi & 86 & - \\
\hline
\end{tabular}

eucalyptus, lemongrass, lemon-scented eucalyptus, amyris, narrow-leaved eucalyptus, carotin, cedarwood, chamomile, cinnamon oil, juniper, cajeput, soya bean, rosemary, niaouli, olive, tagetes, violet, sandalwood, litsea, galbanum, and Curcuma longa also showed good repellency with $8 \mathrm{~h}$ complete repellency against different species of Anopheles genus. Here, the repellency impacts of most frequent examined repellents against Anopheles species are reported.

\section{Citronella}

The repellency effect of citronella was investigated in several studies. Citronella is an essential oil extracted from the stems and leaves of different species of lemongrass (Cymbopogon spp.) [65]. Ansari et al. [11] found that citronella obtained from lemongrass has a $100 \%$ repellency effect against Anopheles culicifacies for $11 \mathrm{~h}$. Amer et al. [6] and Tawatsin et al. [44] also reported that citronella could repel Anopheles stephensi and Anopheles dirus for 8 and $6 \mathrm{~h}$, respectively. Moreover, $100 \mu \mathrm{l}$ and $0.1 \mathrm{ml}$ of citronella grass essential oil showed 2.16 and $0.8 \mathrm{~h}$ complete protection time against $A n$. minimus [45] and An. dirus [47], respectively. The percentage repellency of citronella in other studies. $[6,52,59]$, depending on the concentration of extracts and Anopheles species, was reported to be 52 to $85 \%$.

\section{Peppermint}

Peppermint is a hybrid mint from cross-breeding spearmint (Mentha spicata) and water mint (Mentha aquatica), which contains biologically active constituents and has high menthone, menthol and methyl esters. The plant, indigenous to Europe, is now widespread in cultivation worldwide [66]. The effect of peppermint on Anopheles was explored in 3 studies. Ansari et al. [12] in a field trial revealed that $1 \mathrm{ml}$ peppermint oil without dilution completely repels Anopheles annularis, An. culicifacies and Anopheles subpictus for 11,
9.6 and $7.3 \mathrm{~h}$, respectively and the corresponding percentage repellency were $100 \%, 92.3 \%$ and $83.1 \%$. In another study [6], 20\% oil solutions of peppermint had $57 \%$ repellency and complete protection time for $6.5 \mathrm{~h}$ against An. stephensi. The study by Sritabutra et al. [47] also found that $0.1 \mathrm{ml}$ of peppermint essential oil protect against $A n$. dirus for $1.08 \mathrm{~h}$.

\section{Cinnamomum}

Cinnamomum is a genus in the Laurel family, Lauraceae, several of which are investigated for their antibacterial activity by means of essential oils from bark and leaves [67]. Amer et al. [6] reported that $20 \%$ oil solutions of both camphor (Cinnamomum camphora) and cinnamon (Cinnamomum zeylanicum) had 100\% repellency affect against An. stephensi. While, in the study conducted by Govindarajan et al. [22], C. zeylanicum at $1 \mathrm{mg} / \mathrm{cm}^{2}$ showed $1 \mathrm{~h}$ protection against An. subpictus.

\section{Catnip (Nepeta cataria)}

Catnip is a perennial plant that belongs to the mint family, Labiatae. This herb is spread from central Europe to central Asia and the Iranian plateaus [68]. The 20\% oil solution of catnip in the study carried out by Amer et al. [6], with $100 \%$ protection against An. stephensi for $8 \mathrm{~h}$, had a good effectiveness in preventing Anopheles mosquitoes. Nevertheless, Birkett et al. [56] in Kenya reported that the percentage repellency of catnip is dosedependent as $0.01 \mathrm{mg}, 0.1 \mathrm{mg}$, and $1 \mathrm{mg}$ solutions of this herb had repellency percentage of $17 \%, 97 \%$, and $100 \%$, respectively, against Anopheles gambiae.

\section{Thyme (Thymus serpyllum)}

Thyme is one of nine species belonging to T. serpyllum, a perennial aromatic plant of the Mediterranean flora [69]. Thymus species have been reported to possess 
Table 2 Stratification of potential of plant based repellents

\begin{tabular}{|c|c|c|c|}
\hline Protection time (hours) & Plant name & Concentration/dose & Anopheles species \\
\hline 11.5 & L. sinense ethanolic extract & $25 \%$ & An. minimus \\
\hline 11 & $\begin{array}{l}\text { Pine oil (Pinus) } \\
\text { Citronella (lemongrass oil) } \\
\text { D. sissoo oil } \\
\text { Peppermint oil }\end{array}$ & $\begin{array}{l}1 \mathrm{ml} \text { without dilution } \\
1 \mathrm{ml} \text { without dilution } \\
1 \mathrm{ml} \text { without dilution } \\
1 \mathrm{ml} \text { without dilution }\end{array}$ & $\begin{array}{l}\text { An. culicifacies } \\
\text { An. culicifacies } \\
\text { An. annularis } \\
\text { An. annularis }\end{array}$ \\
\hline $8<$ to $<10$ & $\begin{array}{l}\text { D. sissoo oil } \\
\text { Peppermint oil } \\
\text { R. mucronata oil } \\
\text { R. mucronata oil }\end{array}$ & $\begin{array}{l}1 \mathrm{ml} \text { without dilution } \\
1 \mathrm{ml} \text { without dilution } \\
4 \mathrm{mg} / \mathrm{cm}^{2} \\
3 \mathrm{mg} / \mathrm{cm}^{2}\end{array}$ & $\begin{array}{l}\text { An. culicifacies } \\
\text { An. culicifacies } \\
\text { An. stephensi } \\
\text { An. stephensi }\end{array}$ \\
\hline \multirow[t]{28}{*}{8} & D. sissoo oil & $1 \mathrm{ml}$ without dilution & An. subpictus \\
\hline & Citronella (C. winterianus) essential oils & $20 \%$ oil solution & An. stephensi \\
\hline & Lavender (L. angustifolia) essential oils & $20 \%$ oil solution & An. stephensi \\
\hline & Camphor (C. camphora) essential oils & $20 \%$ oil solution & An. stephensi \\
\hline & Catnip (N. cataria) essential oils & $20 \%$ oil solution & An. stephensi \\
\hline & Geranium (P.graveolens) essential oils & $20 \%$ oil solution & An. stephensi \\
\hline & Jasmine (J.grandiflorum) essential oils & $20 \%$ oil solution & An. stephensi \\
\hline & Broad-leaved eucalyptus (E. dives) essential oils & $20 \%$ oil solution & An. stephensi \\
\hline & Lemongrass (C. citratus) essential oil & $20 \%$ oil solution & An. stephensi \\
\hline & Lemon-scented eucalyptus (E. citriodora) & $20 \%$ oil solution & An. stephensi \\
\hline & Amyris (A. balsamifera) essential oil & $20 \%$ oil solution & An. stephensi \\
\hline & Narrow-leaved eucalyptus (E. radiata) essential oil & $20 \%$ oil solution & An. stephensi \\
\hline & Carotin oil (G. soja) essential oil & $20 \%$ oil solution & An. stephensi \\
\hline & Cedarwood (J. virginiana) essential oil & $20 \%$ oil solution & An. stephensi \\
\hline & Chamomile (A. nobilis) essential oil & $20 \%$ oil solution & An. stephensi \\
\hline & Cinnamon (C. zeylanicum) essential oil & $20 \%$ oil solution & An. stephensi \\
\hline & Juniper (J.communis) essential oil & $20 \%$ oil solution & An. stephensi \\
\hline & Cajeput (M. leucadendron) essential oil & $20 \%$ oil solution & An. stephensi \\
\hline & Soya bean (G. max) essential oil & $20 \%$ oil solution & An. stephensi \\
\hline & Rosemary (R. officinalis) essential oil & $20 \%$ oil solution & An. stephensi \\
\hline & Niaouli (M. quinquenervia) essential oil & $20 \%$ oil solution & An. stephensi \\
\hline & Olive (O. europaea) essential oil & $20 \%$ oil solution & An. stephensi \\
\hline & Tagetes (T. minuta) essential oil & $20 \%$ oil solution & An. stephensi \\
\hline & Violet (V. odorata) essential oil & $20 \%$ oil solution & An. stephensi \\
\hline & Sandalwood (S. album) essential oil & $20 \%$ oil solution & An. stephensi \\
\hline & Litsea (L. cubeba) essential oil & $20 \%$ oil solution & An. stephensi \\
\hline & Galbanum (F.galbaniflua) essential oil & $20 \%$ oil solution & An. stephensi \\
\hline & C. longa essential oil & $25 \%$ & An. dirus \\
\hline \multirow[t]{4}{*}{$7<$ to $<8$} & R. mucronata oil & $2 \mathrm{mg} / \mathrm{cm}^{2}$ & An. stephensi \\
\hline & Thyme (T. serpyllum) essential oils & $20 \%$ oil solutions & An. stephensi \\
\hline & Peppermint oil & $1 \mathrm{ml}$ without dilution & An. subpictus \\
\hline & R. mucronata oil & $1 \mathrm{mg} / \mathrm{cm}^{2}$ & An. stephensi \\
\hline 7 & Lemon (C. limon) essential oil & $20 \%$ oil solution & An. stephensi \\
\hline \multirow[t]{4}{*}{$6<$ to $<7$} & A. monophylla essential oil & 50 ppm & An. stephensi \\
\hline & rosewood (A. rosaeodora) essential oils & $20 \%$ oil solution & An. stephensi \\
\hline & myrtle (M. communis) essential oil & $20 \%$ oil solution & An. stephensi \\
\hline & peppermint (M. piperita) essential oil & $20 \%$ oil solution & An. stephensi \\
\hline
\end{tabular}


Table 2 (continued)

\begin{tabular}{|c|c|c|c|}
\hline Protection time (hours) & Plant name & Concentration/dose & Anopheles species \\
\hline \multirow[t]{6}{*}{6} & Helichrysum (H. italicum) essential oil & $20 \%$ oil solution & An. stephensi \\
\hline & C. martinii martinii var sofia oil & $1 \mathrm{ml}$ without dilution & An. sundaicus \\
\hline & Turmeric (C. longa) volatile oil & $3 \mathrm{ml}$ & An. dirus \\
\hline & Citronella & $3 \mathrm{ml}$ & An. dirus \\
\hline & Hairy basil oil & $3 \mathrm{ml}$ & An. dirus \\
\hline & C. rotundus Linn hexane extract & $10 \%$ & An. stephensi \\
\hline \multirow[t]{10}{*}{$5<$ to $<6$} & I. cairica essential oil & $6 \%$ & An. stephensi \\
\hline & Eucalyptus (E. globulus) essential oils & $20 \%$ oil solution & An. stephensi \\
\hline & Verbena (L. citriodora) essential oil & $20 \%$ oil solution & An. stephensi \\
\hline & Chamomile (C. nobile) essential oil & $20 \%$ oil solution & An. stephensi \\
\hline & C. longa essential oil & $15 \%$ & An. dirus \\
\hline & C. longa essential oil & $20 \%$ & An. dirus \\
\hline & M. charantia essential oil & $6 \%$ & An. stephensi \\
\hline & C. citratus extract & $2 \mathrm{mg} / \mathrm{cm}^{2}$ & An. arabiensis \\
\hline & T. procumbens essential oil & $6 \%$ & An. stephensl \\
\hline & Confertifolin essential oil & 10 ppm & An. stephensi \\
\hline \multirow[t]{4}{*}{5} & Frankincense (B. carteri) essential oil & $20 \%$ oil solution & An. stephensi \\
\hline & Sage (S. sclarea) essential oil & $20 \%$ oil solution & An. stephensi \\
\hline & C. longa essential oil & $10 \%$ & An. dirus \\
\hline & $\begin{array}{l}\text { Confertifolin essential oil } \\
\text { P. tuberosa extract }\end{array}$ & $\begin{array}{l}5 \mathrm{ppm} \\
2 \%\end{array}$ & $\begin{array}{l}\text { An. stephensi } \\
\text { An. stephensi }\end{array}$ \\
\hline $4<$ to $<5$ & $\begin{array}{l}\text { Clove oil } \\
\text { Clove oil } \\
\text { C. citratus extract } \\
\text { Myrtle essential oil } \\
\text { I. cairica essential oil }\end{array}$ & $\begin{array}{l}\text { Cream 20\% } \\
20 \% \mathrm{gel} \\
1 / 5 \mathrm{mg} / \mathrm{cm}^{2} \\
50 \% \\
4 \%\end{array}$ & $\begin{array}{l}\text { An. dirus } \\
\text { An. dirus } \\
\text { An. arabiensis } \\
\text { An. stephensi } \\
\text { An. stephensi }\end{array}$ \\
\hline \multirow[t]{2}{*}{4} & C. longa essential oil & $5 \%$ & An. dirus \\
\hline & P. tuberosa extract & $1.5 \%$ & An. stephensi \\
\hline
\end{tabular}

Stratification of potential of plant based repellents by complete protection times, up to July 2018

various beneficial effects, such as antiseptic, carminative, antimicrobial, and antioxidant properties [70]. The $20 \%$ oil solution of thyme in the study conducted by Amer et al. [6], with $100 \%$ protection against An. stephensi for $7.5 \mathrm{~h}$, had a good effectiveness in preventing Anopheles mosquitoes. Nevertheless, another study [58] reported that the complete protection time of thyme at its maximum concentration (100\%) is $1.7 \mathrm{~h}$ against Anopheles albimanus.

\section{Olive (Olea europaea)}

Olive (O. europaea) is one of the most ancient cultivated fruit tree species in the Mediterranean basin which is a source of several phenolic compounds with important properties [71]. The $20 \%$ oil solution of olive in the study conducted by Amer et al. [6], with a mean percentage of repellency $(71.4 \%)$ and complete protection time against An. stephensi for $8 \mathrm{~h}$, had a good effectiveness in preventing An. stephensi mosquitoes. Karunamoorthi et al. [50] also supported that burning of $25 \mathrm{~g}$ of dried O. europaea, comparable to Amer et al. [6], has a percentage repellency of 79.7 against Anopheles arabiensis.

\section{Eucalyptus}

Eucalyptus is a significant short rotation pulpy woody plant, grown generally in tropical regions [72]. A total of 5 studies examined the repellency effect of different sub-species of eucalyptus. In the laboratory trial by Amer et al. [6], narrow-leaved eucalyptus, lemonscented eucalyptus, and broad-leaved eucalyptus protected against An. stephensi for $8 \mathrm{~h}$, while Eucalyptus globulus complete protection time was reported to be $5.5 \mathrm{~h}$. Auysawasdi et al. [41] used E. globulus essential oil at 5\%, 10\%, 15\%, 20\% and 25\% concentrations against $A n$. dirus. All concentrations of E. globulus provided complete repellency ranging from 1.7 to $3.4 \mathrm{~h}$, depending on the concentration applied. Eucalyptus globulus at $0.1 \mathrm{ml}$ dose in a study [47] repelled $A n$. dirus for $1.58 \mathrm{~h}$. Besides, 
$100 \mu \mathrm{l}$ Eucalyptus citriodora repelled An. minimus for $0.5 \mathrm{~h}$ [45]. In contrast, Seyoum et al. found that lemon eucalyptus extract is not affective against An. gambiae [54].

\section{Myrtle (Myrtus communis)}

Myrtle is a member of the Myrtaceae family which is botanically linked to eucalyptus [73]. In 2 studies, repellency effectiveness of myrtle was investigated. The $20 \%$ oil solution of myrtle in the study conducted by Amer et al. [6], with mean percentage repellency of $42.8 \%$ and complete protection time against An. stephensi for $6.5 \mathrm{~h}$, had a good effectiveness in preventing Anopheles mosquitoes. Tavassoli et al. [62] also supported that myrtle at 50\% concentration repels An. stephensi for $4.36 \mathrm{~h}$.

\section{Basil}

Basil is an annual plant of the Ocimum genus, which belongs to the Lamiaceae family and is used in traditional medicine in many parts of the world [74]. In 6 studies, repellency effectiveness of basil against different Anopheles species was investigated. In the laboratory trial by Amer et al. [6], 20\% oil solution of basil essential oil, with mean percentage repellency of $66.7 \%$, had $100 \%$ protective impact against An. stephensi for $3.5 \mathrm{~h}$. Phasomkusolsil et al. [42] used basil essential oil at 0.02, 0.10, and $0.21 \mathrm{mg} / \mathrm{cm}^{2}$ concentrations against $\mathrm{An}$. dirus. The percentage repellency was dose-response and was reported to be $66 \%, 74 \%$ and $96 \%$, respectively. Basil at $0.1 \mathrm{ml}$ dose in other studies $[47,61]$ repelled Anopheles for $1.5 \mathrm{~h}$ and $0.75 \mathrm{~h}$, whereas, Tawatsin et al. [44] found that hairy basil oil provides $100 \%$ protection against An. dirus for $6 \mathrm{~h}$. In contrast, in the study by Seyoum et al. [54], no remarkable repellency effect against An. gambiae was identified.

\section{Tagetes (Tagetes minuta)}

Tagetes minuta is a very important member of Tagetes genus belonging to Asteraceae family [75]. In 2 studies, repellency effectiveness of tagetes was explored. The $20 \%$ oil solution of T. minuta in the study conducted by Amer et al. [6], with complete protection time for $8 \mathrm{~h}$, had a good effectiveness in preventing against An. stephensi. In contrast, Seyoum et al. found that tagetes extract is not affective against An. gambiae [54].

\section{Neem (Azadirachta indica)}

Neem is a versatile tree broadly grown in tropical areas of India [76]. The repellency effect of Neem against different species of Anopheles was investigated in 2 studies. The $20 \%$ Neem oil in a field trial conducted by Amer et al. [6], with mean percentage repellency $71 \%$ had a complete protection time for $3 \mathrm{~h}$ against An. arabiensis.
Nevertheless, Seyoum et al. found that Neem extract is not affective against An. gambiae [54].

\section{Rosemary (Rosmarinus officinalis)}

Rosemary is an evergreen aromatic shrub with a Mediterranean origin, which belongs to Lamiaceae (Labiatae) family [77]. In 2 studies, repellency effectiveness of rosemary was reported. The $20 \%$ oil solution of rosemary in the study conducted by Amer et al. [6], with $100 \%$ protection against An. stephensi for $8 \mathrm{~h}$, had a good effectiveness in preventing Anopheles mosquitoes. Govindarajan et al. [22] also supported that rosemary at 1, 2.5 and $5 \mathrm{mg} / \mathrm{cm}^{2}$ concentrations completely repels An. subpictus for 1,1 , and $1.5 \mathrm{~h}$, respectively.

\section{Clove (Syzygium aromaticum)}

Clove is a naturally occurring spice which has been shown to possess anti-bacterial, anti-oxidant, antipyretic, anti-candidal, and aphrodisiac activities [78]. The repellency effect of clove against different species of Anopheles was investigated in 6 studies. In the study by Phasomkusolsil et al. [42], clove at 0.02, 0.10 and $0.21 \mathrm{mg} / \mathrm{cm}^{2}$ with a dose-dependent trend, showed $82 \%$, $92 \%$, and $98 \%$ repellency against An. dirus. Barnard et al. [58] used clove essential oil at 25\%, 50\%, $75 \%$, and $100 \%$ concentrations against An. albimanus and found that all concentrations of clove provided complete repellency ranging from 1.25 to $3.55 \mathrm{~h}$, depending on the concentration applied. Consistently, clove at 10\%, 50\%, and $100 \%$ concentrations, with a dose-dependent trend, showed $1.33,2.66$, and $3.5 \mathrm{~h}$ complete repellency against $\mathrm{An}$. dirus [43]. Anopheles dirus was repelled by clove for $1 \mathrm{~h}$ in laboratory conditions in Thailand [47]. Another study [45] reported that clove repels An. minimus for $2 \mathrm{~h}$. Moreover, $20 \%$ gel of clove protected against An. dirus for $4.5 \mathrm{~h} \mathrm{[46].}$ All these findings support that clove can be a considered as moderate repellent.

\section{Orange oil (Citrus sinensis)}

Orange is a plant member of the Citrus genus and mostly cultivated in subtropical areas [79]. The repellency effect of orange against different species of Anopheles was investigated in 4 studies. In the study by Murugan et al. [27], orange extract at 50, 150 and 250,350, and $450 \mathrm{ppm}$ showed $0,0.5,0.5,1.5$ and $2 \mathrm{~h}$ complete protection time repellency (100\%) against An. stephensi, respectively. While, in another study [45], it repelled An. minimus for $0.83 \mathrm{~h}$. Similarly, Sritabutra et al. [47] showed that orange repels An. dirus for $0.83 \mathrm{~h}$. Phasomkusolsi et al. [42] also found that orange at $0.02,0.10$, and $0.21 \mathrm{mg} / \mathrm{cm}^{2}$, with a dose-dependent trend, has $44 \%, 54 \%$, and $84 \%$ repellency against $A n$. dirus, respectively. 


\section{Turmeric (C. longa)}

The medicinal plant turmeric, which is a perennial herb, and a member of Zingiberacae family, is commonly used as a spice in human food [80]. In 3 studies, repellency effectiveness of turmeric was examined. Auysawasdi et al. [41] used turmeric essential oil at 5\%,10\%,15\%,20\%, and $25 \%$ concentrations against An. dirus. All concentrations of turmeric, with a dose-response manner, provided complete repellency ranging from 4 to $8 \mathrm{~h}$, depending on the concentration applied. Other studies also found that turmeric oil repels An. dirus for $6 \mathrm{~h}$ [44] and An. minimus [45] for $1 \mathrm{~h}$.

\section{Discussion}

A high level of insecticide resistance has made because of the chemical control of the pests and vectors. To overcome this problem, it is essential to research for alternative approaches to vector control. The field of herbal repellents is extremely fertile as people demand mosquitoes' repellents that are safe, pleasant to usage and ecologically maintainable. As cost is a significant factor, examination of the use of local florae as repellents is highly suggested. Essential oils and extracts of plants are emerging as potential agents for Anopheles spp. control, with easy-to-administer, low-cost, and risk-free properties. In the present systematic review the highest repellency effect against Anopheles mosquitoes was found from $L$. sinense extract, followed by citronella, pine, $D$. sissoo, peppermint and R. mucronata oils with complete protection time ranging from 9.1 to $11.5 \mathrm{~h}$. Essential oils from plants such as lavender, camphor, catnip, geranium, jasmine, broad-leaved eucalyptus, lemongrass, lemonscented eucalyptus, amyris, narrow-leaved eucalyptus, carotin, cedarwood, chamomile, cinnamon oil, juniper, cajeput, soya bean, rosemary, niaouli, olive, tagetes, violet, sandalwood, litsea, galbanum, and C. longa also showed good repellency with $8 \mathrm{~h}$ complete repellency against different species of Anopheles genus.

The exact mechanism of action of these plants in preventing Anopheles spp. bites has not yet been completely clarified. For citronella, as one of the most explored plant for repellency effect against various mosquitoes, it is reported that active compounds in citronella extract for repelling mosquitoes are eugenol, eucalyptol, camphor, linalool, citral, and citronellal [81]. Some data proposes that these agents interfere with olfactory receptors of mosquitoes [82]. A recent study revealed that An. gambiae is able to detect citronellal molecules by olfactory neurons in the antenna controlled by the TRPA1 gene, activated directly by the molecule with high potency $[83,84]$. Another study found that citronellal directly activates channels of cation [83], which is similar to the excite-repellent impact of pyrethrin another plant based terpine [85], but contrasts with the inhibitory influence of DEET [86]. Although the protection time of citronella oil is shorter than that of DEET. Citronella oil could provide sufficient protection time against mosquitoes. For other plants, the underlying mechanism remains to be elucidated. Possibly, the most important aspect in increasing the permanence of such repellents that are effective but volatile is improving formulations of plant extracts to elevate their longevity through the development of nanoemulsions, improved formulations, and fixatives. While alternative uses such as excite-repellency and spatial activity have also been examined [87].

Some caution is important when interpreting the findings. First, a poorly inspected confounding aspect is the effect of sweating on the effectiveness and protection time of repellents, which are approximately all water-soluble, and this might limits the comparability of repellents. Second, in field trial studies, the number of human volunteers as well as the season during which the trial had been performed differed among the included studies. Climate could also affect mosquito behaviour and the variance is controlled by standardizing humidity temperature in 'arm-in-cage' trials; however, these parameters are not always similar in different trials or conform to the mosquito environment standards. Third, it should be highlighted that some plant compounds are irritating to the skin and/or highly toxic to mammals, and natural does not equate to safe. Thus, plants with potential repellency properties should be tested for their possible unpleasant side effects before introducing as alternative products. Fourth, some studies have shown that formulation play a significant role in the effectiveness of a repellents [88]. However, studies have focused more on the search for active compounds than on optimal formulations $[8,29]$. Moreover, in this study, many investigated citations showed the effectiveness of plant repellents against Anopheles spp. mosquitoes. However, when focusing on Anopheles subspecies, there were only a few publications indicating the efficacy of each plant, which resulted in a difficulty to reach a robust conclusion regarding the best herbal candidates to develop new commercial repellents.

This is another area for additional research. Finally, current studies are difficult to be compared and the repellency effectiveness may also differ among subspecies. Unfortunately, a few studies aimed to compare repellency efficacy of a special plant on subspecies of Anopheles. The heterogeneity in the results of the previous studies might be stem from differences in compound concentrations, application dosages, mosquito species, formulations and the assessment method of repellency, as in some trials the protection time until mosquitoes landed was recorded, whereas in the majority of studies the time until mosquitoes bite was 
considered. Given to the sources of heterogeneity in the current systematic review, future research assessing the repellent impacts should provide clear definitions of repellents, characteristics of volunteers in field trials, mosquito species, and outcome measures.

\section{Conclusion}

The results of this study showed that some plants essential oils and extracts have significant repellent activity against Anopheles spp. mosquitoes. The studies in the last two decades have focused on the search for new natural repellents and some plants displayed good repellent activities, but few natural products have been developed so far $[88,89]$. This review calls for the attention of entomologists and people in the field of mosquito-transmitted diseases for understanding the value and potential position of the plant-derived repellents and their role in disease control.

\section{Abbreviation}

DEET: N, N-diethyl-meta-toluamide.

\section{Acknowledgements}

Not applicable.

\section{Authors' contributions}

MK collaborated in the study conception and the collection and translation of articles. AA performed collecting of articles and writing the manuscript. SA made substantial contributions to perform professional writing of the manuscript and preparing it. SHM assisted in translation, writing and elimination of technical errors. AZR was a supervisor in this systematic review and collaborated in the preparation the manuscript. All authors read and approved the final manuscript.

\section{Funding}

None.

\section{Availability of data and materials}

All data generated or analysed during this study are included in this published article.

\section{Ethics approval and consent to participate}

Not applicable.

\section{Consent for publication}

Not applicable.

\section{Competing interests}

The authors declare that they have no competing interests.

\section{Author details}

${ }^{1}$ Department of Medical Entomology and Vector Control, School of Public Health, Tehran University of Medical Sciences, Tehran, Iran. ${ }^{2}$ Health Research Centre, Lifestyle Institute, Baqiyatallah University of Medical Sciences, Tehran, Iran. ${ }^{3}$ Medical Sciences Research Centre, Ghalib University, Kabul, Afghanistan.

Received: 24 July 2019 Accepted: 8 December 2019

Published online: 21 December 2019

\section{References}

1. Alayo M, Femi-Oyewo M, Bakre L, Fashina A. Larvicidal potential and mosquito repellent activity of Cassia mimosoides extracts. Southeast Asian J Trop Med Public Health. 2015;46:596-601.

2. Karunamoorthi K, Girmay A, Hayleeyesus SF. Mosquito repellent activity of essential oil of Ethiopian ethnomedicinal plant against Afro-tropical malarial vector Anopheles arabiensis. J King Saud Univ Sci. 2014;26:305-10.

3. Karunamoorthi K. The counterfeit anti-malarial is a crime against humanity: a systematic review of the scientific evidence. Malar J. 2014;13:209.

4. Sanghong R, Junkum A, Chaithong U, Jitpakdi A, Riyong D, Tuetun B, et al. Remarkable repellency of Ligusticum sinense (Umbelliferae), a herbal alternative against laboratory populations of Anopheles minimus and Aedes aegypti (Diptera: Culicidae). Malar J. 2015;14:307.

5. Soonwera M. Efficacy of essential oil from Cananga odorata (Lamk.) Hook. f. \& Thomson (Annonaceae) against three mosquito species Aedes aegypti (L.), Anopheles dirus (Peyton and Harrison), and Culex quinquefasciatus (Say). Parasitol Res. 2015;114:4531-43.

6. Amer A, Mehlhorn H. Repellency effect of forty-one essential oils against Aedes, Anopheles, and Culex mosquitoes. Parasitol Res. 2006;99:478.

7. Govindarajan M, Rajeswary M, Arivoli S, Tennyson S, Benelli G. Larvicidal and repellent potential of Zingiber nimmonii (J. Graham) Dalzell (Zingiberaceae) essential oil: an eco-friendly tool against malaria, dengue, and lymphatic filariasis mosquito vectors? Parasitol Res. 2016;115:1807-16.

8. Panneerselvam C, Murugan K. Adulticidal, repellent, and ovicidal properties of indigenous plant extracts against the malarial vector, Anopheles stephensi (Diptera: Culicidae). Parasitol Res. 2013;112:679-92.

9. Govindarajan M, Sivakumar R. Repellent properties of Cardiospermum halicacabum Linn (Family: Sapindaceae) plant leaf extracts against three important vector mosquitoes. Asian Pac J Trop Biomed. 2012;2:602-7.

10. Govindarajan M, Mathivanan T, Elumalai K, Krishnappa K, Anandan A. Ovicidal and repellent activities of botanical extracts against Culex quinquefasciatus, Aedes aegypti and Anopheles stephensi (Diptera: Culicidae). Asian Pac J Trop Biomed. 2011;1:43-8.

11. Ansari M, Mittal P, Razdan R, Sreehari U. Larvicidal and mosquito repellent activities of pine (Pinus longifolia, Family: Pinaceae) oil. J Vector Borne Dis. 2005:42:95.

12. Ansari M, Vasudevan P, Tandon M, Razdan R. Larvicidal and mosquito repellent action of peppermint (Mentha piperita) oil. Bioresource Technol. 2000;71:267-71.

13. Ansari M, Razdan R, Tandon M, Vasudevan P. Larvicidal and repellent actions of Dalbergia sissoo Roxb (F Leguminosae) oil against mosquitoes. Bioresource Technol. 2000;73:207-11.

14. Amerasan D, Murugan K, Kovendan K, Kumar PM, Panneerselvam C, Subramaniam J, et al. Adulticidal and repellent properties of Cassia tora Linn. (Family: Caesalpinaceae) against Culex quinquefasciatus, Aedes aegypti, and Anopheles stephensi. Parasitol Res. 2012;111:1953-64.

15. Baskar K, Sudha V, Nattudurai G, Ignacimuthu S, Duraipandiyan V, Jayakumar M, et al. Larvicidal and repellent activity of the essential oil from Atalantia monophylla on three mosquito vectors of public health importance, with limited impact on non-target zebra fish. Phys Mol Plant Pathol. 2018;101:197-201.

16. Govindarajan M. Larvicidal and repellent activities of Sida acuta Burm. F. (Family: Malvaceae) against three important vector mosquitoes. Asian Pac J Trop Med. 2010;3:691-5.

17. Govindarajan M. Ovicidal and repellent properties of Coccinia indica Wight and Arn (Family: Cucurbitaceae) against three important vector mosquitoes. Eur Rev Med Pharmacol Sci. 2011;15:1010-9.

18. Govindarajan M, Kadaikunnan S, Alharbi NS, Benelli G. Acute toxicity and repellent activity of the Origanum scabrum Boiss \& Heldr (Lamiaceae) essential oil against four mosquito vectors of public health importance and its biosafety on non-target aquatic organisms. Enviro Sci Pollut Res. 2016;23:23228-38.

19. Jeyabalan D, Arul N, Thangamathi P. Studies on effects of Pelargonium citrosa leaf extracts on malarial vector. Anopheles stephensi Liston. Bioresource Technol. 2003;89:185-9.

20. Govindarajan M, Rajeswary M, Sivakumar R. Repellent properties of Delonix elata (L) Gamble (Family: Fabaceae) against malaria vector Anopheles stephensi (Liston)(Diptera: Culicidae). J Saudi Soc Agric Sci. 2015;14:128-33. 
21. Govindarajan M, Sivakumar R. Laboratory evaluation of Indian medicinal plants as repellents against malaria, dengue, and filariasis vector mosquitoes. Parasitol Res. 2015;114:601-12.

22. Govindarajan M. Larvicidal and repellent properties of some essential oils against Culex tritaeniorhynchus Giles and Anopheles subpictus Grassi (Diptera: Culicidae). Asian Pac JTrop Med. 2011;4:106-11.

23. Haldar KM, Ghosh P, Chandra G. Larvicidal, adulticidal, repellency and smoke toxic efficacy of Ficus krishnae against Anopheles stephensi Liston and Culex vishnui group mosquitoes. Asian Pac J Trop Dis. 2014:4:S214-20.

24. Kovendan K, Murugan K, Kumar PM, Thiyagarajan P, William SJ. Ovicidal, repellent, adulticidal and field evaluations of plant extract against dengue, malaria and filarial vectors. Parasitol Res. 2013;112:1205-19.

25. Krishnappa K, Elumalai K, Dhanasekaran S, Gokulakrishnan J. Larvicidal and repellent properties of Adansonia digitata against medically important human malarial vector mosquito Anopheles stephensi (Diptera: Culicidae). J Vector Borne Dis. 2012;49:86.

26. Naine SJ, Devi S. Larvicidal and repellent properties of Streptomyces sp. VITJS4 crude extract against Anopheles stephensi, Aedes aegypti and Culex quinquefasciatus (Diptera: Culicidae). Pol J Microbiol. 2014;63:341-8.

27. Murugan K, Kumar PM, Kovendan K, Amerasan D, Subrmaniam J, Hwang J-S. Larvicidal, pupicidal, repellent and adulticidal activity of Citrus sinensis orange peel extract against Anopheles stephensi, Aedes aegypti and Culex quinquefasciatus (Diptera: Culicidae). Parasitol Res. 2012;111:1757-69.

28. Maheswaran R, Ignacimuthu S. Bioefficacy of essential oil from Polygonum hydropiper L. against mosquitoes, Anopheles stephensi and Culex quinquefasciatus. Ecotoxicol Environ Saf. 2013;97:26-31.

29. Panneerselvam C, Murugan K, Kovendan K, Kumar PM. Mosquito larvicidal, pupicidal, adulticidal, and repellent activity of Artemisia nilagirica (Family: Compositae) against Anopheles stephensi and Aedes aegypti. Parasitol Res. 2012;111:2241-51.

30. Prabhu K, Murugan K, Nareshkumar A, Ramasubramanian N, Bragadeeswaran S. Larvicidal and repellent potential of Moringa oleifera against malarial vector, Anopheles stephensi Liston (Insecta: Diptera: Culicidae). Asian Pac J Trop Biomed. 2011;1:124-9.

31. Almehmadi RM. Oviposition deterrent and skin repellent activities of Artemisia herba alba, Matricharia chamomella and Melia azedarach against Culex quinquefasciatus. Saudi J Biol Sci. 2008;15:1012.

32. Rajkumar S, Jebanesan A. Repellent activity of selected plant essential oils against the malarial fever mosquito Anopheles stephensi. Trop Biomed. 2007:24:71-5

33. Rawani A, Banerjee A, Chandra G. Mosquito larvicidal and biting deterrency activity of bud of Polianthes tuberosa plants extract against Anopheles stephensi and Culex quinquefasciatus. J Commun Dis. 2012;44:79-89.

34. Reegan AD, Kinsalin AV, Paulraj MG, Ignacimuthu S. Larvicidal, ovicidal and repellent activities of marine sponge Cliona celata (Grant) extracts against Anopheles stephensi Liston (Diptera: Culicidae). Asian Pac J Trop Med. 2015;8:29-34

35. Swathi S, Murugananthan G, Ghosh S, Pradeep A. Larvicidal and repellent activities of ethanolic extract of Datura stramonium leaves against mosquitoes. Int J Pharm Phytochem Res. 2012;4:25-7.

36. Das M, Ansari M. Evaluation of repellent action of Cymbopogan martinii martinii Stapf var sofia oil against Anopheles sundaicus in tribal villages of Car Nicobar Island, Andaman \& Nicobar Islands, India. J Vector Borne Dis. 2003;40:100.

37. Singh S, Raghavendra K, Dash A. Evaluation of hexane extract of tuber of root of Cyperus rotundus Linn (Cyperaceae) for repellency against mosquito vectors. J Parasitol Res. 2009;2009:e908085.

38. Kamaraj C, Rahuman AA, Bagavan A, Elango G, Zahir AA, Santhoshkumar T. Larvicidal and repellent activity of medicinal plant extracts from Eastern Ghats of South India against malaria and filariasis vectors. Asian Pac JTrop Med. 2011;4:698-705.

39. Kumar KP, Murugan K, Kovendan K, Kumar AN, Hwang J-S, Barnard DR. Combined effect of seaweed (Sargassum wightii) and Bacillus thuringiensis var israelensis on the coastal mosquito, Anopheles sundaicus. Tamil Nadu India Sci Asia. 2012;38:141-6.

40. Madhiyazhagan P, Murugan K, Kumar AN, Nataraj T. Extraction of mosquitocidals from Ocimum canum leaves for the control of dengue and malarial vectors. Asian Pac J Trop Med. 2014;4:S549-55.
41. Auysawasdi N, Chuntranuluck S, Phasomkusolsil S, Keeratinijakal $\checkmark$. Improving the effectiveness of three essential oils against Aedes aegypti (Linn.) and Anopheles dirus (Peyton and Harrison). Parasitol Res. 2016;115:99-106.

42. Phasomkusolsil S, Soonwera M. Comparative mosquito repellency of essential oils against Aedes aegypti (Linn.), Anopheles dirus (Peyton and Harrison) and Culex quinquefasciatus (Say). Asian Pac J Trop Biomed. 2011;1:S113-8.

43. Trongtokit Y, Rongsriyam Y, Komalamisra N, Apiwathnasorn C. Comparative repellency of 38 essential oils against mosquito bites. Phytother Res. 2005; 19:303-9.

44. Tawatsin A, Wratten SD, Scott RR, Thavara U, Techadamrongsin Y. Repellency of volatile oils from plants against three mosquito vectors. J Vector Ecol. 2001;26:76-82.

45. Phasomkusolsil S, Soonwera M. Insect repellent activity of medicinal plant oils against Aedes aegypti (Linn.), Anopheles minimus (Theobald) and Culex quinquefasciatus Say based on protection time and biting rate. Southeast Asian J Trop Med Public Health. 2010;41:831.

46. Trongtokit Y, Curtis CF, Rongsriyam Y. Efficacy of repellent products against caged and free flying Anopheles stephensi mosquitoes. Southeast Asian J Trop Med Public Health. 2005;36:1423.

47. Sritabutra D, Soonwera M, Waltanachanobon S, Poungjai S. Evaluation of herbal essential oil as repellents against Aedes aegypti (L.) and Anopheles dirus Peyton \& Harrion. Asian Pac J Trop Biomed. 2011;1:S124-8.

48. Tuetun B, Choochote W, Kanjanapothi D, Rattanachanpichai E, Chaithong $U$, Chaiwong P, et al. Repellent properties of celery, Apium graveolens L, compared with commercial repellents, against mosquitoes under laboratory and field conditions. Trop Med Int Health. 2005;10:1190-8.

49. Abiy E, Gebre-Michael T, Balkew M, Medhin G. Repellent efficacy of DEET, MyggA, neem (Azedirachta indica) oil and chinaberry (Melia azedarach) oil against Anopheles arabiensis, the principal malaria vector in Ethiopia. Malar J. 2015;14:187.

50. Karunamoorthi K, Mulelam A, Wassie F. Laboratory evaluation of traditional insect/mosquito repellent plants against Anopheles arabiensis, the predominant malaria vector in Ethiopia. Parasitol Res. 2008;103:529-34.

51. Karunamoorthi K, llango K, Murugan K. Laboratory evaluation of traditionally used plant-based insect repellent against the malaria vector Anopheles arabiensis Patton (Diptera: Culicidae). Parasitol Res. 2010;106:1217-23.

52. Solomon B, Gebre-Mariam T, Asres K. Mosquito repellent actions of the essential oils of Cymbopogon citratus, Cymbopogon nardus and Eucalyptus citriodora: evaluation and formulation studies. J Essent Oil Bearing Plants. 2012;15:766-73.

53. Alwala O, Wanzala W, Inyambukho R, Osundwa E, Ndiege I. Characterization and evaluation of repellent effect of essential oil of Mangifera indica L. from Kenya. J Essent Oil Bearing Plants. 2010;13:85-96.

54. Seyoum A, Pålsson K, Kung'a S, Kabiru E, Lwande W, Killeen G, et al. Traditional use of mosquito-repellent plants in western Kenya and their evaluation in semi-field experimental huts against Anopheles gambiae: ethnobotanical studies and application by thermal expulsion and direct burning. Trans R Soc Trop Med Hyg. 2002;96:225-31.

55. Mayeku W, Omollo N, Odalo O, Hassanali A. Chemical composition and mosquito repellency of essential oil of Conyza newii propagated in different geographical locations of Kenya. Med Vet Entomol. 2014;28:253-6.

56. Birkett MA, Hassanali A, Hoglund S, Pettersson J, Pickett JA. Repellent activity of catmint, Nepeta cataria, and iridoid nepetalactone isomers against Afro-tropical mosquitoes, ixodid ticks and red poultry mites. Phytochemistry. 2011;72:109-14.

57. Innocent E, Hassanali A. Constituents of essential oils from three plant species used in traditional medicine and insect control in Tanzania. J Herb Spice Med Plants. 2014;21:219-29.

58. Barnard DR. Repellency of essential oils to mosquitoes (Diptera: Culicidae). J Med Entomol. 1999;36:625-9.

59. Kweka EJ, Mosha F, Lowassa A, Mahande AM, Kitau J, Matowo J, et al. Ethnobotanical study of some of mosquito repellent plants in north-eastern Tanzania. Malar J. 2008;7:152.

60. De Paula JP, Gomes-Carneiro MR, Paumgartten FJ. Chemical composition, toxicity and mosquito repellency of Ocimum selloi oil. J Ethnopharmacol. 2003;88:253-60. 
61. Nour AH, Elhussein SA, Osman NA, Nour AH. Repellent activities of the essential oils of four Sudanese accessions of basil (Ocimum basilicum L.) against Anopheles mosquito. J Appl Sci. 2009;9:2645-8.

62. Tavassoli M, Shayeghi M, Abai MR, Vatandoost H, Khoobdel M, Salari M, et al. Repellency effects of essential oils of Myrtle (Myrtus communis), Marigold (Calendula officinalis) compared with DEET against Anopheles stephensi on human volunteers. Iranian J Arthropod Borne Dis. 2011;5:10-22

63. Younoussa L, Nukenine EN, Danga SPY, Esimone CO. Repellent activity of the creams formulated from Annona senegalensis and Boswellia dalzielii leaf fractions and essential oils against Anopheles gambiae (Diptera: Culicidae). Asian Pac J Trop Dis. 2016;6:973-8.

64. Konan Y, Sylla M, Doannio J, Traoré S. Comparison of the effect of two excipients (karite nut butter and vaseline) on the efficacy of Cocos nucifera, Elaeis guineensis and Carapa procera oil-based repellents formulations against mosquitoes biting in Ivory Coast. Parasite. 2003;10:181-4.

65. Freeman BC, Beattie GA. An overview of plant defenses against pathogens and herbivores. Plant Health Instructor. 2008;149:1-12.

66. Morehead JA. Efficacy of organic insecticides and repellents against brown marmorated stink bug in vegetables. https://vtechworks.lib. vt.edu/handle/10919/71810. Accessed 28 Mar 2016.

67. Yeh R-Y, Shiu Y-L, Shei S-C, Cheng S-C, Huang S-Y, Lin J-C, et al. Evaluation of the antibacterial activity of leaf and twig extracts of stout camphor tree, Cinnamomum kanehirae, and the effects on immunity and disease resistance of white shrimp, Litopenaeus vannamei. Fish Shellfish Immunol. 2009;27:26-32.

68. Grognet J. Catnip: its uses and effects, past and present. Canadian Vet J. 1990;31:455.

69. Abu-Darwish MS, Abu-Dieyeh ZH, Mufeed B, Al-Tawaha ARM, Al-Dalain SYA. Trace element contents and essential oil yields from wild thyme plant (Thymus serpyllum L.) grown at different natural variable environments, Jordan. J Food Agric Environ. 2009;7:920-4.

70. Prado JM, Leal PF, Meireles MAA, Eds. Comparison of manufacturing cost of thyme extract obtained by supercritical fluid extraction and steam distillation. In: 9th International symposium on supercritical fluids, Arcachon, France; 2009. P. 19.

71. Fabbri A, Hormaza J, Polito V. Random amplified polymorphic DNA analysis of olive (Olea europaea L.) cultivars. J Amer Soc Hort Sci. 1995; 120:538-42.

72. Yasodha R, Sumathi R, Chezhian P, Kavitha S, Ghosh M. Eucalyptus microsatellites mined in silico: survey and evaluation. J Genetic. 2008;87:21-5.

73. Walle M, Walle B, Zerihun L, Makonnen E. Sedative-hypnotic like effect of the essential oil from the leaves of Myrtus communis on mice. Am J Biomed Life Sci. 2014;2:70-7.

74. Miele M, Dondero R, Ciarallo G, Mazzei M. Methyleugenol in Ocimum basilicum L. Cv genovese gigante. J Agric Food Chem. 2001;49:517-21.

75. Sadia S, Khalid S, Qureshi R, Bajwa AA. Tagetes minuta L., a useful underutilized plant of family Asteraceae: a review. Pak J Weed Sci Res. 2013;19:179-89.

76. Bahuguna V. Silviculture and management practices for cultivation of Azadirachta indica (Neem). Indian Forester. 1997;123:379-86.
77. Lara M, Gutierrez J, Timon M, Andrés A. Evaluation of two natural extracts (Rosmarinus officinalis L. and Melissa officinalis L.) as antioxidants in cooked pork patties packed in MAP. Meat Sci. 2011;88:481-8.

78. Singh AK, Dhamanigi SS, Asad M. Anti-stress activity of hydro-alcoholic extract of Eugenia caryophyllus buds (clove). Indian J Pharmacol. 2009;41:28.

79. Acar Ü, Kesbiç OS, Yılmaz S, Gültepe N, Türker A. Evaluation of the effects of essential oil extracted from sweet orange peel (Citrus sinensis) on growth rate of tilapia (Oreochromis mossambicus) and possible disease resistance against Streptococcus iniae. Aquaculture. 2015;437:282-6.

80. Durrani F, Ismail M, Sultan A, Suhail S, Chand N, Durrani Z. Effect of different levels of feed added turmeric (Curcuma longa) on the performance of broiler chicks. J Agric Biol Sci. 2006;1:9-11.

81. Moore S, Lenglet A, Hill N. Plant-based insect repellents. In: Debboun M, Frances SP, Strickman D, editors. Insect repellents: principles, methods and uses. 1st ed. Boca Raton: CRC Press; 2006. p. 275-304.

82. Pappenberger B, Geier M, Boeckh J. Responses of antennal olfactory receptors in the yellow fever mosquito Aedes aegypti to human body odours. In: Foundation Ciba, editor. Olfaction in mosquito-host interactions. Chichester: Wiley; 1996. p. 254-66.

83. Kwon Y, Kim SH, Ronderos DS, Lee Y, Akitake B, Woodward OM, et al. Drosophila TRPA1 channel is required to avoid the naturally occurring insect repellent citronellal. Curr Biol. 2010;20:1672-8.

84. Kongkaew C, Sakunrag I, Chaiyakunapruk N, Tawatsin A. Effectiveness of citronella preparations in preventing mosquito bites: systematic review of controlled laboratory experimental studies. Trop Med Int Health. 2011;16:802-10.

85. Soderlund DM, Bloomquist JR. Neurotoxic actions of pyrethroid insecticides. Annu Rev Entomol. 1989;34:77-96.

86. Ditzen M, Pellegrino M, Vosshall LB. Insect odorant receptors are molecular targets of the insect repellent. DEET Sci. 2008;319:1838-42.

87. Tyagi B. Advances in vector mosquito control technologies, with particular reference to herbal products. In: Veer V, Gopalakrishnan R, editors. Herbal insecticides, repellents and biomedicines: effectiveness and commercialization. New Delhi: Springer; 2016. p. 1-9.

88. Lupi E, Hatz C, Schlagenhauf P. The efficacy of repellents against Aedes, Anopheles, Culex and Ixodes spp. A literature review. Trav Med Infect Dis. 2013;11:374-411.

89. Yogananth N, Anuradha V, Ali MYS, Muthezhilan R, Chanthuru A, Prabu MM. Chemical properties of essential oil from Rhizophora mucronata mangrove leaf against malarial mosquito Anopheles stephensi and filarial mosquito Culex quinquefasciatus. Asian Pac J Trop Med. 2015;5(suppl 1):S67-72.

\section{Publisher's Note}

Springer Nature remains neutral with regard to jurisdictional claims in published maps and institutional affiliations.

\footnotetext{
Ready to submit your research? Choose BMC and benefit from:

- fast, convenient online submission

- thorough peer review by experienced researchers in your field

- rapid publication on acceptance

- support for research data, including large and complex data types

- gold Open Access which fosters wider collaboration and increased citations

- maximum visibility for your research: over $100 \mathrm{M}$ website views per year
}

At BMC, research is always in progress.

Learn more biomedcentral.com/submissions 